\title{
In medium properties of an axion within a $2+1$ flavor Polyakov loop enhanced Nambu-Jona-Lasinio model
}

\author{
Aman Abhishek, ${ }^{1, *}$ Arpan Das $\oplus^{2, \dagger}{ }^{2,}$ Ranjita K. Mohapatra, ${ }^{3, \ddagger}$ and Hiranmaya Mishra $\oplus^{1, \S}$ \\ ${ }^{1}$ Theory Division, Physical Research Laboratory, Navrangpura, Ahmedabad 380 009, India \\ ${ }^{2}$ Institute of Nuclear Physics Polish Academy of Sciences, PL-31-342 Krakow, Poland \\ ${ }^{3}$ Department of Physics, Banki Autonomous College, Cuttack 754008, India
}

(Received 6 July 2020; revised 5 February 2021; accepted 8 March 2021; published 6 April 2021)

\begin{abstract}
We estimate in medium properties of axion i.e., its mass and self-coupling within a three flavor Polyakov loop extended Nambu-Jona-Lasinio (PNJL) model with Kobayashi-Maskawa-t'Hooft determinant interaction. We also estimate the topological susceptibility of strong interaction within the same model. It is observed that (statistical) confinement effects simulated by Polyakov loop potential play an important role in the estimation of all these quantities, particularly, near the critical temperature. Both the mass and the self-coupling of the axion get correlated with the chiral and deconfinement transition. The results for all these quantities obtained within the PNJL model is compared with chiral perturbation theory, NambuJona-Lasinio (NJL) model and lattice QCD simulation results wherever available. The results for properties of axions at finite baryon densities are also presented.
\end{abstract}

DOI: 10.1103/PhysRevD.103.074003

\section{INTRODUCTION}

The axion was originally introduced to solve the strong $C P$ (charge conjugation and parity) problem in a dynamical way [1-4]. Due to the non-Abelian nature of the gauge fields, QCD allows the topologically nontrivial ChernSimons term, $\mathcal{L}_{\theta} \sim \theta \operatorname{Tr} G_{\mu \nu} \tilde{G}^{\mu \nu}$. This Chern-Simons term, which is allowed by the gauge symmetry, does not affect the classical equation of motion. However, this term has important quantum mechanical consequences [5]. For a nonvanishing value of $\theta, C P$ symmetry is explicitly broken in QCD. Stringent constraints on the $C P$-violating $\theta$ term comes from the measurement of the electric dipole moment $(\mathrm{EDM})$ of the neutron, i.e., $\theta \lesssim 10^{-11}[6,7]$. The smallness of $\theta$ implied by the EDM constraint is a fine-tuning problem involving a precise cancellation between two dimensionless terms generated by physics at different scales corresponding to QCD and weak interaction scales. Spontaneous breaking of the Peccei-Quinn (PQ) symmetry is an elegant mechanism to solve the strong $C P$ problem in a dynamical manner which predicts the smallness of

\footnotetext{
*aman@prl.res.in

†arpan.das@ifj.edu.pl

ranjita.iop@gmail.com

shm@prl.res.in
}

Published by the American Physical Society under the terms of the Creative Commons Attribution 4.0 International license. Further distribution of this work must maintain attribution to the author(s) and the published article's title, journal citation, and DOI. Funded by SCOAP ${ }^{3}$. the $\theta[1,2]$. Spontaneous breaking of Peccei-Quinn (PQ) symmetry also naturally predicts a pseudo-Goldstone boson which is known as the axion [8,9]. In the original axion model formulated by Peccei and Quinn [1,2], Weinberg [3] and Wilczek [4], the spontaneous breaking of the $U(1)_{\mathrm{PQ}}$ symmetry occurs simultaneously with the electroweak symmetry breaking giving rise to observational signals of axions which is in contradiction with the observational evidence, e.g., $K, J / \psi$ meson decay [10]. These constraints can be avoided in the invisible axion models where the PQ symmetry breaking occurs at a higher scale giving rise to a very light and weakly interacting axion [11-14].

Axion mass and the coupling to the other particles are inversely proportional to the PQ symmetry breaking scale. Hence axions are very light as well as weakly interacting particles and have been considered as a candidate for dark matter [15-22]. The effect of the axion on the stellar evolution has also been considered in the literature. Due to the strangeness-changing nonleptonic weak interaction during the conversion of a neutron star into a strange star, axions could be copiously produced. Axions might also drastically alter the energy budget of stars [23]. Axions can be produced in hot and dense astrophysical plasma which can transport energy out of stars. One can put a strong constraint on the axion properties i.e., mass, coupling with normal matter and radiation using the stellar-evolution lifetimes or energy-loss rates observation [24-27]. For stellar longevity, the rate at which a star can liberate its nuclear-free energy is important. It is not only controlled by the nuclear reaction rates but also depends on the rate at 
which nuclear energy can be transported through the star and radiated into the vacuum. In the absence of weakly interacting low mass (as compared to typical stellar temperature) particles, the energy is dissipated away from stars by photons. However, a weakly interacting low mass particle has the potential to efficiently transport energy away thereby shortening the lifetime of stars. Note that for efficient transport of energy liberated in the nuclear reactions in a star, the particle should be weakly interacting, but the interaction with the nuclear matter should not be very small so that the weakly interacting particles should be produced in sufficient numbers to carry away the nuclear energy efficiently. For stars hotter than about $10^{8}$ Kelvin neutrino cooling becomes comparable with the photon cooling. Being a small mass particle and weakly interacting in nature, axions, if produced in the hot and dense medium inside the stars can also act as a coolant. In stars the axions are produced by the Compton-like process $\left(\gamma+e^{-} \rightarrow\right.$ $\left.a+e^{-}\right)$, the Primakoff process $\left(\gamma+Z\left(e^{-}\right) \rightarrow a+Z\left(e^{-}\right)\right)$ and axion bremsstrahlung $\left(e^{-}+Z \rightarrow a+e^{-}+Z\right)$. The axion emissivity due to these processes is proportional to the axion mass $\left(m_{a}^{2}\right)$ [19]. Therefore estimation of axion mass is of great importance to investigate the effects of the axion on the stellar cooling. Further it has been suggested that axions can form stars as well as a Bose-Einstein condensate [28-40]. In the context of Bose-Einstein condensation of dark matter axions, to estimate the thermalization of axion, its self-interaction plays an important role in the calculation of the relaxation rate [41]. Furthermore, ultralight axion self-interactions can play an important role in the large scale structure of the Universe [42]. It is therefore of paramount importance to know the characteristics of axion properties, e.g., axion mass and the selfcoupling in a hot and dense medium.

It is important to note that there are various calculations of finite temperature axion mass available in literature using, for instance, dilute instanton gas, lattice QCD and instanton liquid model and which may not agree with one another [43-48]. The temperature dependence of axion mass is important as it can affect significantly the axion dark matter abundance. Estimation of the axion potential closer to the QCD transition scale is also important and in the absence of this knowledge, one parametrizes the axion mass in a way that resembles the result from the dilute instanton gas model $[49,50]$. It may also be noted that such a parametrization of the axion mass with temperature can have a discontinuity that does not encapsulate the variation of axion mass across the QCD transition scale [51]. At relatively high temperatures with respect to the quark hadron transition scale, one can use perturbative techniques to estimate axion properties, e.g., the dilute instanton gas approximation, however around and below the QCD transition scale, nonperturbative effects can have a significant impact on the axion mass and coupling. To estimate the response of the axion to a QCD thermal medium one can use QCD inspired effective field theories and phenomenological models as, for instance, chiral perturbation theory $(\chi \mathrm{PT})$ [52-54] and the Nambu-Jona-Lasinio (NJL) model $[55,56] . \chi \mathrm{PT}$, which has been used to study the $\theta$ vacuum of QCD and QCD axion physics, predicts a value of topological susceptibility which agrees with the lattice QCD results at zero temperature [57-63]. Further, the computation of the axion potential can be extended to finite temperature for chiral Lagrangian. In particular, at temperatures below the QCD transition scale $(\sim 170 \mathrm{MeV})$ using chiral Lagrangian the temperature dependence of the axion potential and its mass can be estimated. Although the $\chi \mathrm{PT}$ can give a reliable prediction at low temperature, at high temperature $\chi \mathrm{PT}$ results may not be reliable, because in $\chi \mathrm{PT}$ there are no partonic degrees of freedom which becomes important near and above the QCD transition temperature. Perturbative expansion is also not under control around the QCD transition scale and nonperturbative methods are required to study the axion properties.

Because of the limitation of $\chi \mathrm{PT}$ and perturbative techniques one can use QCD inspired chiral effective models, e.g., the Nambu-Jona-Lasinio (NJL) model to investigate the thermal properties of the axion. The NJL model has been used earlier to study the $C P$-violating effects and the effect of the theta vacuum on the QCD phase diagram [64-69]. This approach has been considered in Refs. [70,71] to study the axion mass and self-coupling at finite temperature in the absence as well as in the presence of a magnetic field. All these calculations show that near the chiral transition temperature the axion mass and self-coupling are significantly modified. The NJL model which effectively explains one of the key features of QCD, i.e., chiral symmetry breaking and its restoration, does not address the effects of the gluon degrees of freedom adequately. In the NJL model the gluonic degrees of freedom are replaced by a local four-Fermi-type interaction of quark color currents. Improvements upon the NJL model, e.g., the Polyakov loop enhanced Nambu-JonaLasinio (PNJL) model takes into account this missing feature by including a temporal background gluon field in a manner that the single quark states below the transition temperature are statistically suppressed. As a result, both chiral and deconfinement aspects of QCD are captured within a single framework [72-79]. In the PNJL model, the nonzero value of the Polyakov loop around the QCD transition scale plays an important role, which may be important to study the axion physics, particularly across the QCD transition scale.

Therefore, in the present article, we study the axion mass and self-coupling using the three flavor Polyakov loop enhanced Nambu-Jona-Lasinio (PNJL) model. The two most important properties of QCD are the chiral transition and confinement-deconfinement transition. Hence for an effective description of the QCD medium near the transition temperature, the effective model should also reflect 
these properties of QCD. The Nambu-Jona-Lasinio (NJL) model which is a key ingredient of the PNJL model only deals with the fermionic part without gauge fields. The NJL model includes the global symmetries of QCD in the fermionic sector such as chiral symmetry, baryon number, electric charge and strange number symmetries. In the NJL model, the dynamical generation of mass due to the multiquark interactions leads to the spontaneous breaking of chiral symmetry. In the NJL model, the local $S U(3)_{c}$ gauge symmetry of QCD is replaced by a global $S U(3)_{c}$ symmetry. So the NJL model lacks the confinement property due to the absence of the QCD gauge fields. Note that for $N_{c}=2$ confinement is less significant and the thermodynamics can be described quite successfully using the simplest NJL model [80]. On the other hand for $N_{c}=3$ confinement is significant. In the PNJL model both the chiral condensate and the Polyakov loop are considered as classical homogeneous fields which couple to the quarks according to the symmetries and symmetry breaking patterns of QCD, therefore describing various aspects of confinement and chiral symmetry breaking in a unifying framework. The confinement-deconfinement transition which is characterized by the Polyakov loop order parameter is well defined in the static quark limit. The confinement-deconfinement transition is characterized by the spontaneous breaking of the $Z(3)$ center symmetry of QCD [81-84]. However, in the presence of dynamical quarks, the center symmetry is explicitly broken. Hence in the presence of a dynamical quark the Polyakov loop cannot be considered as an order parameter, but the Polyakov loop still serves as an indicator of the confinement-deconfinement transition [85-90]. It is important to note that the PNJL model also has some limitations, e.g., in the PNJL model the Polyakov loop is considered as a simple static background field. Furthermore, transverse gluons, which play an important role in the thermodynamics of QCD matter at a very high temperature $T \gtrsim 2.5 T_{c}$, are not considered in the PNJL model. Therefore the PNJL model is expected to describe QCD thermodynamics only within a limited range of temperature [91]. The previous studies of axion properties within the framework of the NJL model indicate that QCD transition significantly modifies axion mass and self-coupling [70,71]. Axion mass and axion self-coupling decrease rapidly across the chiral transition temperature and there is a correlation between the quark-antiquark condensate and axion properties studied in this model. In the PNJL model at finite temperature the presence of the nonvanishing value of the Polyakov loop affects the quark-antiquark condensate, which eventually also affects the axion mass and its self-coupling at finite temperature.

This paper is organized in the following manner. After the introduction, in Sec. II we discuss the formalism to study the axion properties, i.e., its mass and self-coupling in the three flavor PNJL model. Using the formalism as given in Sec. II we estimate the axion mass and self-coupling at finite temperature and quark chemical potential. We show the results and the discussions of these results in Sec. III. Finally in Sec. IV we conclude our investigation with an overview of it.

\section{FORMALISM: AXION WITHIN THE PNJL MODEL}

QCD, in principle, can have a parity violating term, the so-called $\theta$ term,

$$
\mathcal{L}_{\theta}=\frac{\theta g_{s}^{2}}{64 \pi^{2}} G_{\mu \nu}^{a} \tilde{G}_{a}^{\mu \nu}
$$

where $G_{\mu \nu}^{a}$ is the gluon field strength and $\tilde{G}_{\mu \nu}^{a}$ its dual. Such term respects Lorentz invariance as well as gauge invariance but violates parity unless $\theta=0 \bmod \pi$. However, in nature, $\mathrm{QCD}$ respects $C P$ to a large extent in vacuum, as is indicated by the fact that the magnitude of the $C P$-violating term, $\theta$, arising from the measurement of the intrinsic electric dipole moment of neutrons, is small, $\theta<0.7 \times 10^{-11}$.

A dynamical and elegant way to explain the smallness of $\theta$ is to elevate $\theta$ to a field in such a way that it has a vanishing vacuum expectation value. The normalized axion field is denoted as $a(x)=\theta(x) f_{a}$. Here $f_{a}$ is the axion decay constant which also represents the PQ symmetry breaking scale. The phenomenology of the axion is controlled by the axion decay constant $f_{a}$. Astrophysical observations, e.g., cooling rate of the SN1987A supernova and black hole superradiance put stringent bounds on the PQ symmetry breaking scale, $10^{8} \lesssim f_{a} \lesssim 10^{17} \mathrm{GeV}$ [92-97]. Typically one could consider $f_{a}$ to be of the order of the grand unified scale (GUT scale) $\sim 10^{16} \mathrm{GeV}$. Hence the interaction between the axion field and the QCD gauge field now can be expressed as $\mathcal{L}_{\theta} \propto\left(a / f_{a}\right) G \tilde{G}$. In vacuum for $\theta=0$, spontaneous parity violation does not exist as per the Vafa-Witten theorem [98]. On the other hand, there could be $C P$ violation for $\theta=\pi$, by the Dashen phenomena, with the appearance of two degenerate $C P$-violating vacua separated by a potential barrier. Because of the nonperturbative nature of this $C P$-violating term, this has been studied in different low energy effective models. In the present investigation, we shall confine our attention to the PNJL model.

The PNJL model which is an extension of the NJL model is defined by a Lagrangian which couples the quarks to a temporal background gauge field representing Polyakov loop dynamics. Explicitly, the Lagrangian density of the three flavor PNJL model with the Kobayashi-Maskawa't Hooft determinant interaction term incorporating the interaction with the axion can be expressed as $[67,99]$ 


$$
\begin{aligned}
\mathcal{L}= & \bar{q}\left(i \gamma^{\mu} D_{\mu}-\hat{m}\right) q+G_{s} \sum_{A=0}^{8}\left[\left(\bar{q} \lambda^{A} q\right)^{2}+\left(\bar{q} i \gamma_{5} \lambda^{A} q\right)^{2}\right] \\
& -K\left[e^{i \theta} \operatorname{det}\left\{\bar{q}\left(1+\gamma^{5}\right) q\right\}+e^{-i \theta} \operatorname{det}\left\{\bar{q}\left(1-\gamma^{5}\right) q\right\}\right] \\
& +\mathcal{U}(\Phi, \bar{\Phi}, T) .
\end{aligned}
$$

Here $q=\left(q_{u}, q_{d}, q_{s}\right)^{T}$ is the quark field, and $\hat{m}$ represents the current quark mass matrix $\operatorname{diag}\left(m_{u}, m_{d}, m_{s}\right)$. In the present investigation we consider $m_{u}=m_{d}=m_{0}$. $\lambda^{0}=\sqrt{2 / 3} I_{3 \times 3}$; here $I_{3 \times 3}$ is the $3 \times 3$ identity matrix in flavor space, $\lambda^{A}$ with $A=1,2, \ldots, 8$ are the Gell-Mann matrices in flavor space. The covariant derivative $D^{\nu}=$ $\partial^{\nu}-i A^{\nu}$ and $A^{\nu}=\delta_{0}^{\nu} A^{0}$. The gauge coupling is absorbed in the definition of $A_{\mu}=g_{s} \mathcal{A}_{\mu}^{A} \frac{\lambda^{A}}{2}$, where $\mathcal{A}_{\mu}^{A}$ is the $\mathrm{SU}(3)$ gauge field and $\lambda_{A}$ are the Gell-Mann matrices, $g_{s}$ is the gauge coupling. In the NJL sector, $G_{s}$ denotes the coupling of the four-quark interaction which includes scalar and pseudoscalar type interactions. This interaction term is symmetric under $S U(3)_{L} \times S U(3)_{R} \times U(1)_{V} \times U(1)_{A} \times$ $S U(3)_{C}$ symmetry. $K$ is the coupling of the KobayashiMaskawa-'t Hooft determinant interaction. This determinant is taken in the flavor space. The determinant interaction term explicitly breaks the $U(1)_{A}$ symmetry of the Lagrangian. The Polyakov loop potential $\mathcal{U}(\Phi, \bar{\Phi}, T)$ is the effective potential of the traced Polyakov loop and its Hermitian conjugate,

$$
\Phi=\frac{1}{N_{c}} \operatorname{Tr} L, \quad \bar{\Phi}=\frac{1}{N_{c}} \operatorname{Tr} L^{\dagger} .
$$

This trace is in the color space. The Polyakov loop operator $L$ is the Wilson loop in the temporal direction which can be expressed as [100,101]

$$
L(\vec{x})=\mathcal{P} \exp \left[i \int_{0}^{\beta} d \tau A_{0}(\vec{x}, \tau)\right], \quad \beta=1 / T,
$$

where $\mathcal{P}$ is a path ordering operator in the imaginary time $\tau=i t$. In a gauge where $A^{0}$ is time independent, one can perform the integration trivially and we will have $L=\exp \left(i \beta A_{0}\right)$. Further, one can rotate the gauge field in the Cartan subalgebra $A_{0}^{c}=A_{0}^{3} \lambda^{3}+A_{0}^{8} \lambda^{8}$, so that $L$ is diagonal in the color space [102].

In the absence of quarks, the Polyakov loop can be considered as an order parameter for the confinementdeconfinement transition. The confinement-deconfinement transition is characterized by the spontaneous breaking of the $Z(3)$ center symmetry of QCD. For vanishing chemical potential $\Phi=\bar{\Phi}$, but at finite baryon chemical potential in general $\Phi \neq \bar{\Phi}$. At low temperatures, the Polyakov loop potential has a unique minimum at $\Phi=0=\bar{\Phi}$. However, at a higher temperature, above the transition temperature, an absolute minimum of $\mathcal{U}$ occurs at a nonvanishing value of $\Phi$ and $\bar{\Phi}$. For vanishing baryon chemical potential, in the
TABLE I. Parameters for Polyakov loop potential.

\begin{tabular}{lccccc}
\hline \hline$a_{0}$ & $a_{1}$ & $a_{2}$ & $a_{3}$ & $b_{3}$ & $b_{4}$ \\
\hline 6.75 & -1.95 & 2.625 & -7.44 & 0.75 & 7.5 \\
\hline \hline
\end{tabular}

high-temperature limit $T \rightarrow \infty, \Phi \rightarrow 1$. The chosen effective potential $\mathcal{U}$ which is written in terms of $\Phi$ and $\bar{\Phi}$, following the $Z(3)$ symmetry is expressed as

$\mathcal{U}(\Phi, \bar{\Phi}, T)=\left[-\frac{b_{2}(T)}{2} \bar{\Phi} \Phi-\frac{b_{3}}{6}\left(\Phi^{3}+\bar{\Phi}^{3}\right)+\frac{b_{4}}{4}(\Phi \bar{\Phi})^{2}\right] T^{4}$,

with

$$
b_{2}(T)=a_{0}+a_{1}\left(\frac{T_{0}}{T}\right)+a_{2}\left(\frac{T_{0}}{T}\right)^{2}+a_{3}\left(\frac{T_{0}}{T}\right)^{3} .
$$

The coefficients $a_{i}$ and $b_{i}$ and $T_{0}$ can be fixed using the pure-gauge lattice QCD data. The coefficients $a_{i}$ and $b_{i}$ are given in Table I. The critical temperature $T_{0}$ for confinement-deconfinement phase transition is fixed to be $270 \mathrm{MeV}$ in the pure gauge sector [72-75]. However in the presence of fermions the value for $T_{0}$ changes as discussed in Ref. [103]. Accordingly in the present case of $(2+1)$ flavors, we have taken $T_{0}=187 \mathrm{MeV}$.

In the mean-field approximation the thermodynamic potential $(\Omega)$ of the PNJL model at finite temperature $(T)$ and quark chemical potential $(\mu)$ can be expressed as $[65-67,99]$

$$
\begin{aligned}
& \Omega\left(\sigma_{i}, \eta_{i}, \Phi, \bar{\Phi}, \theta, T, \mu\right) \\
&=\Omega_{\bar{q} q}+\sum_{i} 2 G_{s}\left(\sigma_{i}{ }^{2}+\eta_{i}{ }^{2}\right)+4 K\left(\cos \theta \sigma_{u}^{2} \sigma_{s}+\sin \theta \eta_{u}^{2} \eta_{s}\right) \\
&-4 K\left(\cos \theta\left(2 \sigma_{u} \eta_{u} \eta_{s}+\eta_{u}{ }^{2} \sigma_{s}\right)\right. \\
&\left.+\sin \theta\left(2 \sigma_{u} \sigma_{s} \eta_{u}+\sigma_{u} \sigma_{u} \eta_{s}\right)\right)+\mathcal{U}(\Phi, \bar{\Phi}, T) .
\end{aligned}
$$

Here $\sigma_{i}=-\left\langle\bar{q}_{i} q_{i}\right\rangle$ is the scalar condensate for the flavor $i(i=u, d, s)$ and $\eta_{i}=-\left\langle\bar{q}_{i} i \gamma_{5} q_{i}\right\rangle$ is the pseudoscalar condensate for flavor $i(i=u, d, s)$. In the mean field approximation, the fields are replaced by their expectation (thermal expectation) values. One may note here that we have neglected the contribution of flavor off diagonal terms arising from determinant interaction to the thermodynamic potential, as such a contribution arising from the off diagonal terms are $1 / N_{c}$ suppressed compared to the diagonal terms [65,66]. However off diagonal terms become relevant in the presence of a finite isospin chemical potential, as has been considered in Refs. [104,105]. In the present investigation, we confine our attention to the case of symmetric matter where all the quarks have the same chemical potential. Throughout the manuscript, we have 
used the notation $\sigma, \eta, \Phi$ and $\bar{\Phi}$ to represent the fields as well as their expectation values for simplicity and convenience. As mentioned above at a low temperature where $\Phi=0$ and $\bar{\Phi}=0$, the potential has only one minimum. For temperatures higher than the transition temperature, the $\Phi$ and $\bar{\Phi}$ develop a nonvanishing vacuum expectation value, and the cubic term in the Polyakov loop potential leads to
$Z$ (3) degenerate vacua. Generally for $a=0$ or $\theta=0$ the pseudoscalar condensate $\eta_{i}$ vanishes. Therefore parity, as well as $C P$, is not broken at $\theta=0$. But any nonvanishing value of $\eta$ indicates the breaking of parity symmetry and $C P$ violation. In the PNJL model the fermionic contribution to the thermodynamic potential in the grand canonical ensemble is

$$
\begin{aligned}
\Omega_{\bar{q} q}= & -2 N_{c} \sum_{i} \int \frac{d \mathbf{p}}{(2 \pi)^{3}} E_{p}^{i}-2 T \sum_{i} \int \frac{d \mathbf{p}}{(2 \pi)^{3}}\left(\log \left[1+3 \Phi \exp \left[-\beta \omega_{-}^{i}\right]+3 \bar{\Phi} \exp \left[-2 \beta \omega_{-}^{i}\right]+\exp \left[-3 \beta \omega_{-}^{i}\right]\right]\right. \\
& \left.+\log \left[1+3 \bar{\Phi} \exp \left[-\beta \omega_{+}^{i}\right]+3 \Phi \exp \left[-2 \beta \omega_{+}^{i}\right]+\exp \left[-3 \beta \omega_{+}^{i}\right]\right]\right) .
\end{aligned}
$$

In the above $N_{c}=3$ is the number of colors, $i$ is the flavor index, $\beta=1 / T$ is the inverse of temperature, $\omega_{ \pm}^{i}=E_{p}^{i} \pm \mu$ is the excitation energy for antiquarks and quarks respectively. Here $E_{p}^{i}$ is the single-particle energy for flavor $i$ given as

$$
E_{p}^{i}=\sqrt{\mathbf{p}^{2}+M^{i 2}}, \quad M^{i}=\sqrt{M_{s}^{i 2}+M_{p s}^{i 2}},
$$

where $M^{i}$ is the constituent quark mass arising from the scalar and pseudoscalar condensates. Further, $M_{s}^{i}$ and $M_{p s}^{i}$ can be expressed in terms of the scalar and pseudoscalar condensates in the following manner:

$$
\begin{aligned}
M_{s}^{u}= & M_{s}^{d} \\
=m_{u} & +4 G_{s} \sigma_{u}+2 K\left(\cos \theta\left(\sigma_{u} \sigma_{s}-\eta_{u} \eta_{s}\right)\right. \\
- & \left.\sin \theta\left(\sigma_{u} \eta_{s}+\eta_{u} \sigma_{s}\right)\right) \\
M_{p s}^{u}= & M_{p s}^{d} \\
= & 4 G_{s} \eta_{u}-2 K\left(\cos \theta\left(\sigma_{u} \eta_{s}+\eta_{u} \sigma_{s}\right)\right. \\
& \left.+\sin \theta\left(\sigma_{u} \sigma_{s}-\eta_{u} \eta_{s}\right)\right),
\end{aligned}
$$

and

$M_{s}^{s}=m_{s}+4 G_{s} \sigma_{s}+2 K\left(\cos \theta\left(\sigma_{u}^{2}-\eta_{u}^{2}\right)-2 \sin \theta \sigma_{u} \eta_{u}\right)$,

$M_{p s}^{s}=4 G_{s} \eta_{s}-2 K\left(2 \cos \theta \sigma_{u} \eta_{u}+\sin \theta\left(\sigma_{u}^{2}-\eta_{u}^{2}\right)\right)$.

The $\sigma_{i}$ and $\eta_{i}$ are scalar and pseudoscalar condensates respectively for flavor $i$ and are given as

$$
\sigma_{i}=-\left\langle\bar{q}_{i} q_{i}\right\rangle=2 N_{c} \int \frac{d \mathbf{p}}{(2 \pi)^{3}} \frac{M_{s}^{i}}{M^{i}}\left(1-f_{p}\left(\omega_{-}^{i}\right)-f_{a p}\left(\omega_{+}^{i}\right)\right),
$$

$$
\begin{aligned}
\eta_{i} & =-\left\langle\bar{q}_{i} i \gamma_{5} q_{i}\right\rangle \\
& =2 N_{c} \int \frac{d \mathbf{p}}{(2 \pi)^{3}} \frac{M_{p s}^{i}}{M^{i}}\left(1-f_{p}\left(\omega_{-}^{i}\right)-f_{a p}\left(\omega_{+}^{i}\right)\right) .
\end{aligned}
$$

Here $f_{p}$ and $f_{a p}$ are the thermal distribution functions for the quarks and the antiquarks respectively which get modified by the Polyakov loop as

$$
\begin{aligned}
f_{p}\left(\omega_{-}^{i}\right) & =\frac{\Phi e^{-\beta \omega_{-}^{i}}+2 \bar{\Phi} e^{-2 \beta \omega_{-}^{i}}+e^{-3 \beta \omega_{-}^{i}}}{1+3\left(\Phi+\bar{\Phi} e^{-\beta \omega_{-}^{i}}\right) e^{-\beta \omega_{-}^{i}}+e^{-3 \beta \omega_{-}^{i}}}, \\
f_{a p}\left(\omega_{+}^{i}\right) & =\frac{\bar{\Phi} e^{-\beta \omega_{+}^{i}}+2 \Phi e^{-2 \beta \omega_{+}^{i}}+e^{-3 \beta \omega_{+}^{i}}}{1+3\left(\bar{\Phi}+\Phi e^{-\beta \omega_{+}^{i}}\right) e^{-\beta \omega_{+}^{i}}+e^{-3 \beta \omega_{+}^{i}}} .
\end{aligned}
$$

Minimizing the thermodynamic potential with respect to scalar $\left(\sigma^{i}\right)$ and pseudoscalar $\left(\eta^{i}\right)$ condensates as well as the Polyakov loop variable $(\Phi)$ and its conjugate $(\bar{\Phi})$ results in the following gap equations:

$$
\frac{\partial \Omega}{\partial \sigma^{i}}=0 ; \quad \frac{\partial \Omega}{\partial \eta^{i}}=0 ; \quad \frac{\partial \Omega}{\partial \Phi}=0 ; \quad \frac{\partial \Omega}{\partial \bar{\Phi}}=0 .
$$

The first two gap equations lead to the self-consistent equation for $M_{s}^{i}$ and $M_{p s}^{i}$ as in Eqs. (10)-(13). The last two gap equations lead to the following two self-consistent equations for the Polyakov loop variables as

$$
\begin{aligned}
& I_{\Phi}+T^{4}\left(-\frac{b_{2}}{2} \bar{\Phi}-\frac{b_{3}}{2} \Phi^{2}+\frac{b_{4}}{2} \Phi \bar{\Phi}^{2}\right)=0 \\
& I_{\bar{\Phi}}+T^{4}\left(-\frac{b_{2}}{2} \Phi-\frac{b_{3}}{2} \bar{\Phi}^{2}+\frac{b_{4}}{2} \bar{\Phi} \Phi^{2}\right)=0 .
\end{aligned}
$$


Here we have defined

$$
\begin{aligned}
I_{\Phi} & =\frac{\partial \Omega_{\bar{q} q}}{\partial \Phi} \\
& =\sum_{i}-6 T \int \frac{d \mathbf{p}}{(2 \pi)^{3}}\left[\frac{e^{-\beta \omega_{-}^{i}}}{1+3 \Phi e^{-\beta \omega_{-}^{i}}+3 \bar{\Phi} e^{-2 \beta \omega_{-}^{i}}+e^{-3 \beta \omega \omega_{-}^{i}}}+\frac{e^{-2 \beta \omega_{+}^{i}}}{1+3 \bar{\Phi} e^{-\beta \omega_{+}^{i}}+3 \Phi e^{-2 \beta \omega_{+}^{i}}+e^{-3 \beta \omega_{+}^{i}}}\right] \\
I_{\bar{\Phi}} & =\frac{\partial \Omega_{\bar{q} q}}{\partial \bar{\Phi}} \\
& =\sum_{i}-6 T \int \frac{d \mathbf{p}}{(2 \pi)^{3}}\left[\frac{e^{-2 \beta \omega_{-}^{i}}}{1+3 \Phi e^{-\beta \omega_{-}^{i}}+3 \bar{\Phi} e^{-2 \beta \omega_{-}^{i}}+e^{-3 \beta \omega_{-}^{i}}}+\frac{e^{-\beta \omega_{+}^{i}}}{1+3 \bar{\Phi} e^{-\beta \omega_{+}^{i}}+3 \Phi e^{-2 \beta \omega_{+}^{i}}+e^{-3 \beta \omega_{+}^{i}}}\right] .
\end{aligned}
$$

The fermionic contribution to the thermodynamic potential of the PNJL model as given in Eq. (8) involves a vacuum contribution $(T=0, \mu=0)$ and a medium contribution $(T \neq 0, \mu \neq 0)$. The vacuum term is ultraviolet (UV) divergent. Various regularization methods have been used in the literature to regulate this vacuum term, for instance, a sharp three momentum cutoff, and the proper time regularization scheme. In this investigation we consider a sharp three momentum cutoff $(\Lambda)$ to regulate the vacuum term. In the medium-term distribution function takes care of the ultraviolet problem.

The parameters used in the NJL part are the same as in Refs. $[65,66]$. The parameters are fixed by fitting the model to zero temperature pion mass, pion decay constant and masses of kaon and $\eta^{\prime}$. Here the cutoff parameter is $\Lambda=602.3 \mathrm{MeV}$, the bare quark masses are taken as $m_{u}=m_{d}=5.5 \mathrm{MeV}, m_{s}=140.7 \mathrm{MeV}$ while the couplings are given as $G_{s} \Lambda^{2}=1.835$ and the determinant coupling $K \Lambda^{5}=12.36$. With this parametrization, one gets a constituent quark mass for up and down quarks of $M_{u, d}=$ $368 \mathrm{MeV}$ and for strange quark of $M_{s}=549 \mathrm{MeV}$ at zero temperature and vanishing chemical potential. With this set of parameters, the mass of $\eta$ is underestimated by 6 percent.

Let us note that after solving the gap equation for a given $T$ and $\mu$ with all the condensates determined, the thermodynamic potential as given in Eq. (7) gives rise to the axion effective potential $\tilde{\Omega}(\theta, T, \mu)$ as a function of $\theta \equiv a / f_{a}$ within the PNJL model:

$$
\begin{aligned}
& \tilde{\Omega}(\theta, T, \mu)=\Omega\left[\sigma^{i}(\theta, T, \mu), \eta^{i}(\theta, T, \mu), \Phi(\theta, T, \mu),\right. \\
& \bar{\Phi}(\theta, T, \mu), \theta, T, \mu] \text {. }
\end{aligned}
$$

Using the axion potential one can obtain the axion mass and the axion self-coupling can be obtained as [70]

$$
m_{a}^{2}=\left.\frac{d^{2} \tilde{\Omega}}{d a^{2}}\right|_{a=0}=\frac{\chi}{f_{a}^{2}} ; \quad \lambda_{a}=\left.\frac{d^{4} \tilde{\Omega}}{d a^{4}}\right|_{a=0} .
$$

Here $\chi$ is the topological susceptibility given by the second derivative of effective potential with respect to $\theta$.
Note that all the physical condensates $\sigma^{i}, \eta^{i}, \Phi$ and $\bar{\Phi}$ have implicit dependence on the axion field, so that

$\frac{d \tilde{\Omega}}{d a}=\frac{\partial \tilde{\Omega}}{\partial a}+\frac{\partial \tilde{\Omega}}{\partial \sigma^{i}} \frac{\partial \sigma^{i}}{\partial a}+\frac{\partial \tilde{\Omega}}{\partial \eta^{i}} \frac{\partial \eta^{i}}{\partial a}+\frac{\partial \tilde{\Omega}}{\partial \Phi} \frac{\partial \Phi}{\partial a}+\frac{\partial \tilde{\Omega}}{\partial \bar{\Phi}} \frac{\partial \bar{\Phi}}{\partial a}$.

Therefore to evaluate axion mass and self-coupling as given in Eq. (22) we have to evaluate $\frac{\partial^{(n)} \sigma^{i}}{\partial a^{(n)}}, \frac{\partial^{(n)} \eta^{i}}{\partial a^{(n)}}, \frac{\partial^{(n)} \Phi}{\partial a^{(n)}}$ and $\frac{\partial^{(n)} \bar{\Phi}}{\partial a^{(n)}}$, where $n=1,2,3,4$ represents the order of the derivative. $\frac{\partial^{(n)} \sigma^{i}}{\partial a^{(n)}}, \frac{\partial^{(n)} \eta^{i}}{\partial a^{(n)}}, \frac{\partial^{(n)} \Phi}{\partial a^{(n)}}$ and $\frac{\partial^{(n)} \bar{\Phi}}{\partial a^{(n)}}$ can be evaluated by taking the successive derivative of the gap equations as given in Eq. (18), with respect to the axion field $a$.

\section{RESULTS AND DISCUSSION}

\section{A. Vanishing quark chemical potential}

In this subsection, we present the results for vanishing quark chemical potential $\mu=0$ for which the Polyakov loop $\Phi$ and its conjugate $\bar{\Phi}$ are the same. In general, the fields $\Phi$ and $\bar{\Phi}$ are different only at nonvanishing quark chemical potential.

As may be noted from the thermodynamic potential of the PNJL model, at temperatures below the critical temperature, the Polyakov loop has a negligible value, and the quark excitations are highly suppressed statistically. Only for temperatures near $T_{c}$ and above, the Polyakov loop attains a significant value to affect the quark condensates. In fact, due to the nonvanishing values of $\Phi$ the chiral transition temperature in the PNJL model is higher with respect to the same in the NJL model. In what follows, we refer to NJL model results which are obtained by replacing $\Phi=\bar{\Phi}=1$ in Eq. (8) and taking $\mathcal{U}(\Phi, \bar{\Phi}, T)=0$ in Eq. (7). Thus, we are taking the same parameters in the NJL and PNJL model in the quark sector for comparison.

For a given temperature $T$, and chemical potential $\mu$ and a $C P$-violating parameter $\theta$, we solve the coupled selfconsistent Eqs. (10)-(20). Since we have assumed isospin symmetry for the light quarks ( $u$ and $d$ quarks), we have six coupled gap equations: two for scalar condensate related to the masses $M_{s}^{u}$ and $M_{s}^{s}$, two for pseudoscalar condensate 
related to $M_{p s}^{u}$ and $M_{p s}^{s}$ and two for Polyakov loop variables $\Phi$ and $\bar{\Phi}$. The solutions to these equations are then substituted in Eq. (7) and are then checked whether they correspond to global minima of thermodynamic potential or not. If there are multiple solutions of gap equations, we choose the one for which the thermodynamic potential is minimum.

In Fig. 1 we have shown the condensates for $u$ and $s$ quarks and also the Polyakov loop variable as a function of temperature for $\theta=0$ in Fig. 1(a) and for $\theta=2 \pi / 3$ in Fig. 1(b). We have normalized the quark condensates with respect to their values at zero temperature. We note that while solving the gap equations (10)-(13) and (18)-(20), we have used the cutoff $\Lambda$ while considering the contributions from the terms independent of the medium while we have considered the cutoff to be infinity for the medium dependent part. Further, the Polyakov loop at a higher temperature beyond the critical temperature slightly exceeds unity. Such an observation is also there in Ref. [103]. This is a reflection of the parametrization of the Polyakov loop potential. A different parametrization using the logarithmic function of fields avoids Polyakov loop

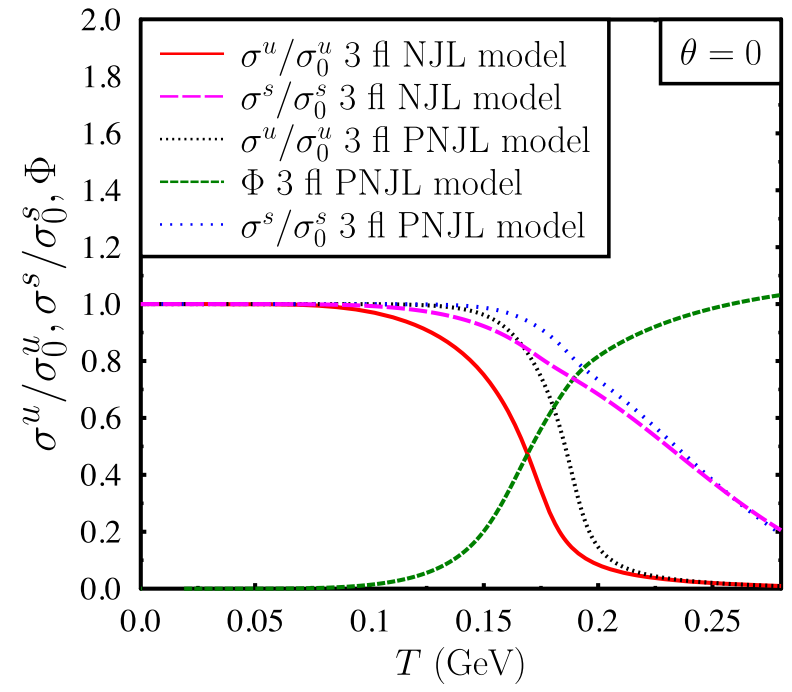

(a)

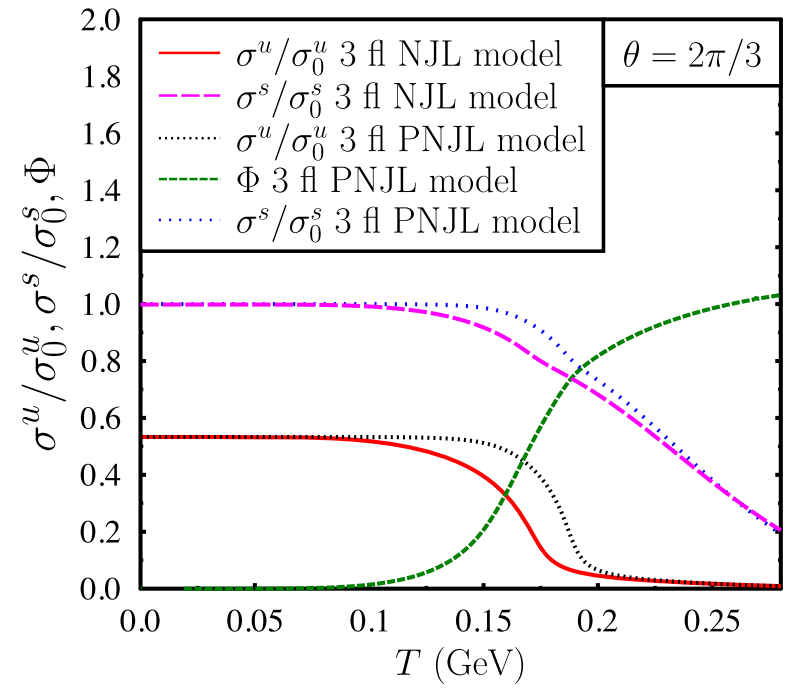

(b)

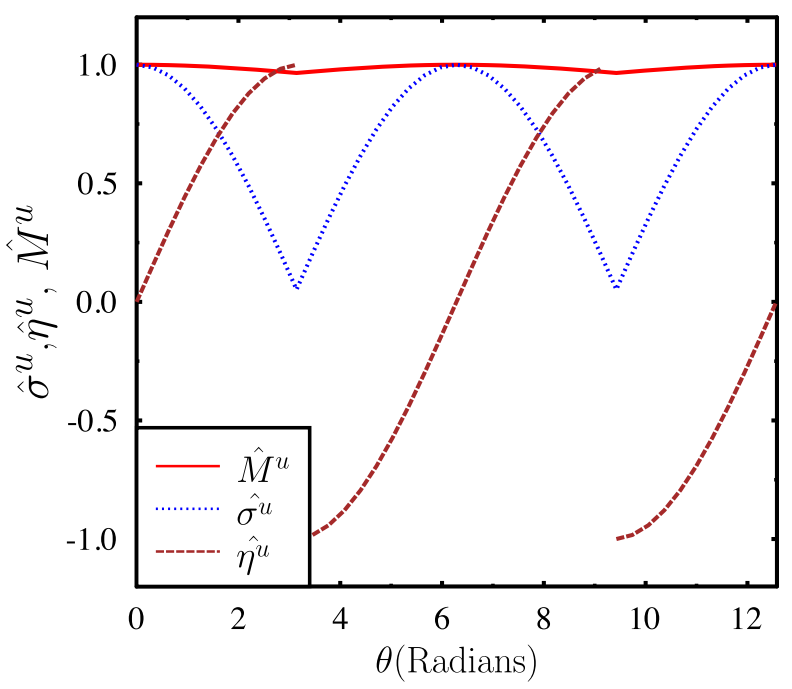

(c)

FIG. 1. (a) Variations of the normalized scalar condensates $\sigma^{u} / \sigma_{0}^{u}, \sigma^{s} / \sigma_{0}^{s}$ and $\Phi$ with temperature for $\theta=0$. (b) Variations of the normalized scalar condensates $\sigma^{u} / \sigma_{0}^{u}, \sigma^{s} / \sigma_{0}^{s}$ and $\Phi$ with temperature for $\theta=2 \pi / 3$. Here $\sigma_{0}^{i}$ denotes the scalar condensate in vacuum for $\theta=0$ for flavor $i$. (c) Plot of the normalized scalar condensate $\left(\hat{\sigma}_{u}\right)$, pseudoscalar condensate $\left(\hat{\eta}_{u}\right)$ and the normalized constituent mass $\left(\hat{M}_{u}\right)$ as a function of $\theta . M_{u}$ and $\sigma_{u}$ are normalized to their values in vacuum $(T=0)$ at $\theta=0$ while $\eta^{u}$ is normalized to its value in vacuum $(T=0)$ at $\theta=\pi$. 
value going beyond unity $[106,107]$. As may be noted in Fig. 1(a), at low temperatures up to $100 \mathrm{MeV}$, the medium effects are small and the light quark condensate ratio remains close to its zero temperature value of unity. Beyond $100 \mathrm{MeV}$, the Polyakov loop becomes nonvanishing and suppresses statistically the medium contribution for the PNJL model as compared to the NJL model resulting in a higher critical temperature for chiral crossover transition. Further, the strange quark condensate values remain significant beyond the temperature when the chiral symmetry for the light quarks is almost restored.

As may be observed in Fig. 1(b), the values of the light quark scalar condensates for $\theta=2 \pi / 3$ decrease compared to their values for $\theta=0$ by almost half. A similar change is not observed for the strange condensate whose value remains almost constant for $\theta=0$ and $\theta=2 \pi / 3$. This is due to the fact that strange pseudoscalar condensate $\left(\eta^{s}\right)$ is very small in magnitude (to be discussed later). However, for all the condensates, the temperature dependence remains similar to the $\theta=0$ case. This is because the temperature dependence is decided by the quark mass which depends on both the scalar and pseudoscalar condensates and remains almost constant with respect to variation in $\theta$ as seen in Eq. (9). This is seen in Fig. 1(c) where we have shown the variation of the normalized scalar and pseudoscalar condensates as well as the constituent quark mass at $T=0 \mathrm{MeV}$ as a function of $\theta$. The value of the constituent quark mass and the scalar condensates are normalized to their corresponding values at $\theta=0$, while the pseudoscalar condensate is normalized to its value at $\theta=\pi$. From Fig. 1(c) it may be noted that the

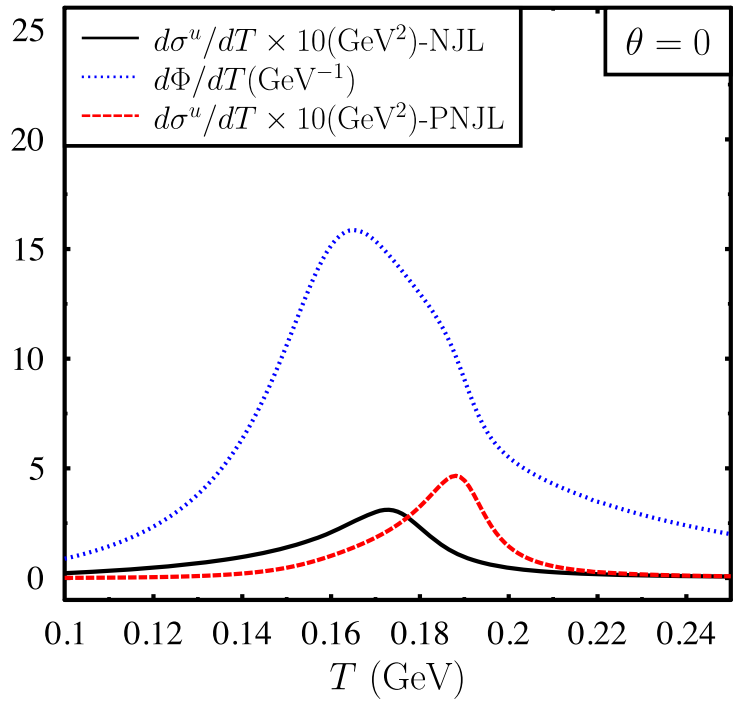

(a) scalar and pseudoscalar condensates vary in a complementary manner such that constituent quark mass remains almost constant. It may also be observed that from Figs. 1(a) and 1(b) the Polyakov loop parameter remains almost unchanged compared to its value at $\theta=0$. The reason is that the Polyakov loop does not couple directly with $\theta$, but gets affected indirectly by the condensates $\sigma$ and $\eta$ which themselves depend upon the $C P$-violating parameter $\theta$. The gap equation for the Polyakov loop variables, as given in Eqs. (19) and (20), are obtained by differentiating thermodynamic potential with respect to Polyakov loop variables. In doing so, the contribution arises from $\Omega_{\bar{q} q}$ and $\mathcal{U}(\Phi, \bar{\Phi})$, both of which are almost independent of $\theta$ variation. $\Omega_{\bar{q} q}$ depends on $\sigma$ and $\eta$ through the quark mass $M$, which as we have seen is almost independent of $\theta$. Therefore the Polyakov loop parameter is almost independent of $\theta$. We, however, note that there is a weak dependence of the Polyakov loop parameter on $\theta$ as we shall discuss later.

Since the transition is a crossover here, we define the transition temperature as the maximum in the derivative of the order parameters. This is shown in Fig. 2. In Fig. 2(a), we show the variation of the derivatives of the order parameters, i.e., $d \sigma^{u} / d T$ for the NJL and the PNJL model and $d \Phi / d T$ for the PNJL model. We have multiplied the quark condensate derivative by a factor of 10 so as to compare them with the variation of $d \Phi / d T$ in the same plot. Figure 2(a) is for $\theta=0$ and Fig. 2(b) is for $\theta=2 \pi / 3$. From Fig. 2 we can see that the variation of $d \sigma^{u} / d T$ and $d \Phi / d T$ shows a nonmonotonic behavior with temperature with a peak. The peak in the variation of $d \sigma^{u} / d T$ and

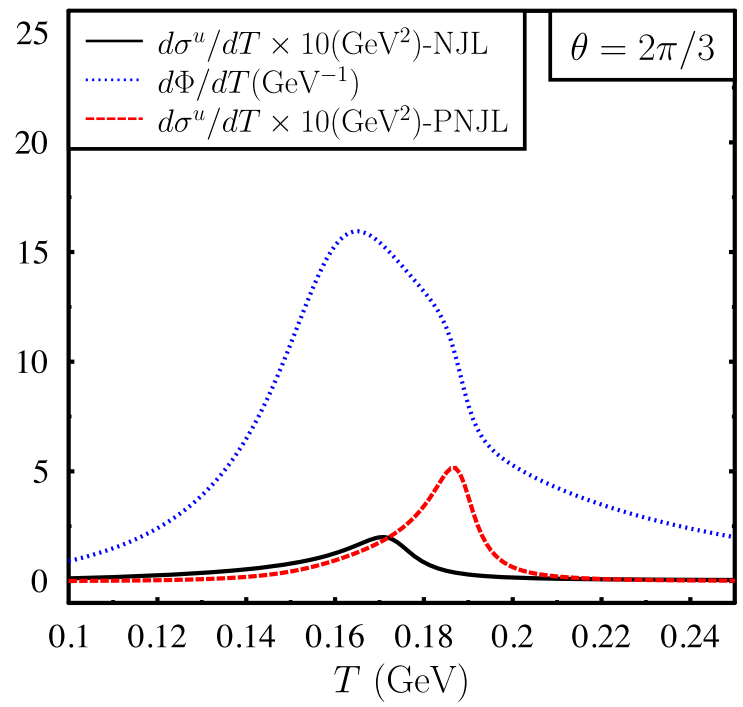

(b)

FIG. 2. Left plot: variation of $d \sigma^{u} / d T$ and $d \Phi / d T$ with temperature in the PNJL and NJL model for $\theta=0$. Peak structure in the variation of $d \sigma^{u} / d T$ indicates that the location of the pseudocritical temperature in the NJL model is $T_{c \sigma}=173 \mathrm{MeV}$, while in the PNJL model $T_{c \sigma}=188 \mathrm{MeV}$. The peak in the variation of $d \Phi / d T$ for PNJL model is at $T_{c \Phi}=166 \mathrm{MeV}$. Right plot: variation of $d \sigma^{u} / d T$ and $d \Phi / d T$ with temperature in the PNJL and NJL model for $\theta=2 \pi / 3$. 
$d \Phi / d T$ indicates the chiral transition temperature $\left(T_{c \sigma}\right)$ and the confinement-deconfinement transition temperature $\left(T_{c \Phi}\right)$ respectively. The critical temperature for deconfinement is $T_{c \Phi}=166 \mathrm{MeV}$ while $T_{c \sigma}=188 \mathrm{MeV}$ for the PNJL model. It may also be noted that at the crossover transition the Polyakov loop value is about 0.65 as compared to its asymptotic value $\Phi \sim 1$. Similarly, the scalar condensate value at the peak of the corresponding susceptibility $T_{c \sigma}$ is $\sigma\left(T_{c \sigma}\right) \equiv 0.597 \sigma_{0}$. Nonvanishing condensates near $T_{c}$ indicate importance of nonperturbative effects. It is clear from Fig. 2 that the chiral transition temperature is higher in the PNJL model as compared to the

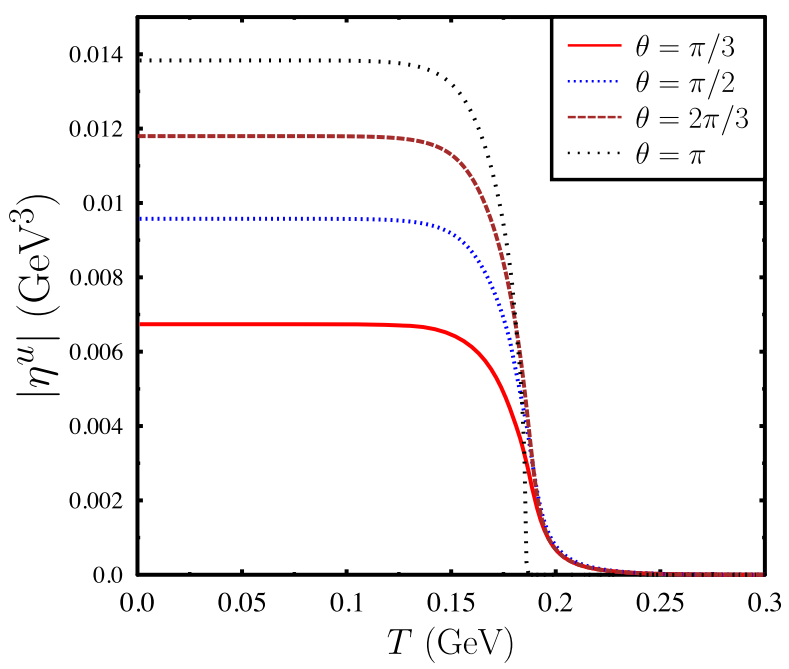

(a)
NJL model. The chiral and deconfinement transition temperature are slightly different as may be noted from the peak position and differ by about $20 \mathrm{MeV}$. As noted earlier, this temperature behavior is the same for $\theta=2 \pi / 3$ as shown in the right plot of Fig. 2 due to negligible change in constituent mass with $\theta$.

Next, we discuss in Fig. 3 the behavior of the magnitude of pseudoscalar condensates $\eta^{i}$ with temperature. Let us note that $\left|\eta^{i}\right|$ vanishes for $\theta=0$ and is maximum for $\theta=\pi$ (below the $C P$ restoration temperature). The behavior of $\left|\eta^{i}\right|$ for different temperatures is shown in Fig. 3(a). The behavior is similar to the scalar condensate as a function of
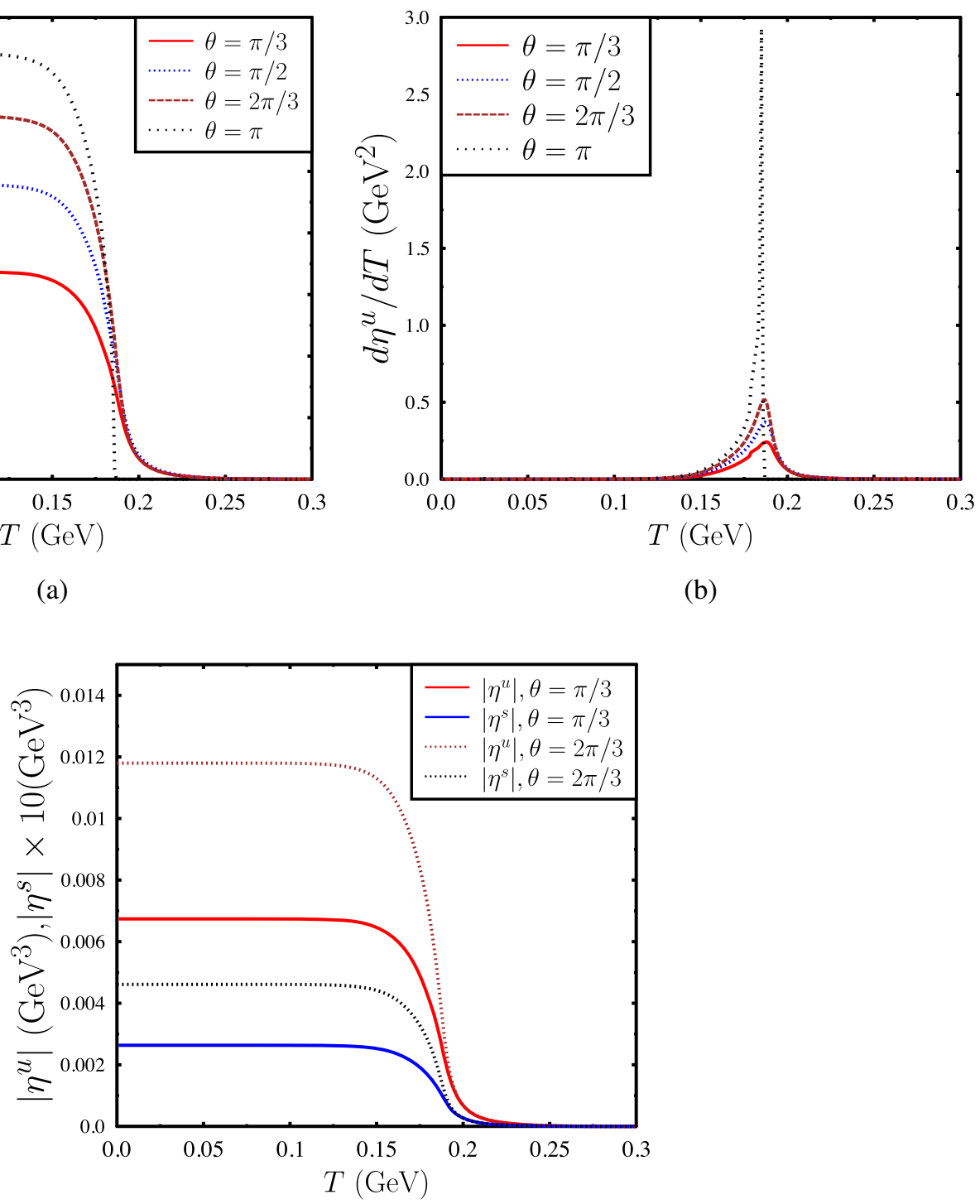

(b)

(c)

FIG. 3. (a) and (b) show variation of the magnitude of pseudoscalar condensate $\left(\left|\eta^{u}\right|\right)$ and its derivative $\left(d \eta^{u} / d T\right)$ respectively with temperature for $\theta=\pi / 3, \pi / 2,2 \pi / 3, \pi$ in a three flavor PNJL model. Nonvanishing value of the pseudoscalar condensate indicates the breaking of the parity symmetry $(\mathrm{P})$ or equivalently the breaking of the $C P$ (charge conjugation and parity) symmetry in QCD. At zero temperature $\eta$ has nonvanishing value for nonzero $\theta$, but at a high enough temperature the $\eta$ condensate vanishes. In (c) we compare the magnitude of pseudoscalar condensates for $u$ and $s$ quarks. 
temperature as shown in Fig. 1(a). As may be observed, the transition is a crossover for all values of $\theta$ except for $\theta=\pi$ where the pseudoscalar condensate vanishes at high temperature leading to a second order transition [65,66]. Similar to the chiral transition and deconfinement transition one can also define $C P$ transition temperature from the corresponding peak in $d \eta^{u} / d T$ which we have plotted in Fig. 3(b). As $\theta$ increases the peak value of $d \eta^{u} / d T$ increases. The critical temperature for $C P$ transition is $\sim 188 \mathrm{MeV}$, the same as for the chiral transition temperature. For $\theta=\pi$ the pseudoscalar condensate vanishes beyond $T_{c}$, however, the constituent quark mass still remains above current quark mass due to the finite value for the scalar condensates. Such high temperature restoration of $C P$ is expected as the instanton effects that are responsible for $C P$ violation become suppressed exponentially [108]. However, it should be noted that nonequilibrium processes such as heavy ion collision can have local $C P$ violation that can be induced by sphaleron processes which are not suppressed at high $T$ [109].

In Fig. 3(c) we plotted the magnitude of pseudoscalar condensate $|\eta|$ for up and strange quarks as a function of temperature. It turns out that the parity violating pseudoscalar condensate due to the strange quarks is about an order of magnitude less than light quark pseudoscalar condensates. Such a flavor violation for the pseudoscalar condensate could be related to the large mass of the strange quarks compared to the light quarks. This is in contrast to the scalar condensates which is discussed later.

In Fig. 4 we have shown the variation of $\sigma^{u}$ with respect to $\theta$ at three different temperatures. We have chosen here the temperatures below and above $T_{c} \sim 188 \mathrm{MeV}$

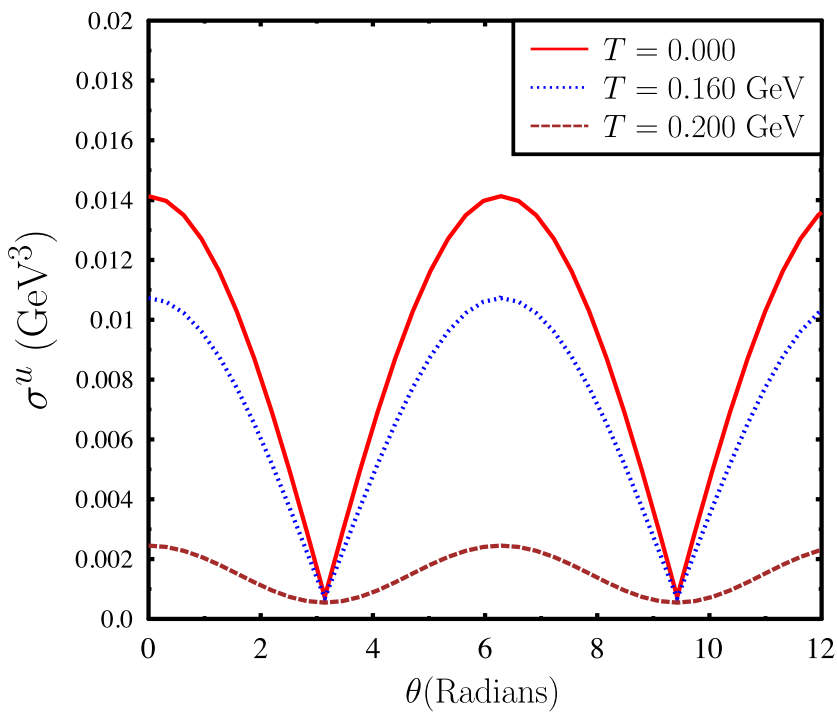

FIG. 4. Variation of scalar condensate $\sigma^{u}$ with $\theta$ for three different values of temperature in the PNJL model. The periodic variation of $\sigma^{u}$ with $\theta$ is due to the dependence of the thermodynamic potential on the periodic functions $\sin (\theta)$ and $\cos (\theta)$. including the zero temperature, i.e., $T=0,160$ and $200 \mathrm{MeV}$. The periodic behavior of the condensate with respect to $\theta$ is due to $\cos \theta$ and $\sin \theta$ dependent terms present in the thermodynamic potential. This behavior of the condensate with $\theta$ is similar to the results as obtained in the NJL model in Refs. [65,66,70,71]. $\sigma^{u}$ reaches its maximum value for $\theta=2 i \pi$, for $i=0,1,2 \ldots$ etc. and attains minimum for $\theta=(2 i+1) \pi$ at all temperatures.

Figure 5 represents the variation of the pseudoscalar condensate $\eta^{u}$ with respect to $\theta$ for different values of temperatures. Similar to Fig. 4 here also we consider values of temperature below and above the transition temperature. As may be observed the pseudoscalar condensate behaves in a complementary manner to the scalar condensate as a function of $\theta$ as mentioned earlier. At $T=0, \eta^{u}$ is discontinuous for $\theta=(2 i+1) \pi$, for $i=0,1,2 \ldots$ etc. and vanishes for $\theta=2 i \pi$. At $T=0, \eta^{u}$ starts with a vanishing value and increases in magnitude monotonically with an increase in $\theta$ up to $\theta=\pi$ at which point it changes discontinuously to a value equal in magnitude but opposite in sign and then decreases monotonically in magnitude and vanishes at $\theta=2 \pi$. At $\theta=\pi$ there exist two degenerate vacua which are in agreement with the Dashens phenomena [110]. The two vacua which have opposite signs of the condensate $\eta$ differ by a $C P$ transformation between them. It may be noted that the effective potential as a function of $\theta$ is maximum at $\theta=\pi$ (as discussed later). This degeneracy for $\eta^{u}$ at $\theta=\pi$ is lifted beyond $T_{c}=188 \mathrm{MeV}$ as seen in Fig. 3. Hence Dashen's phenomenon breaks down beyond

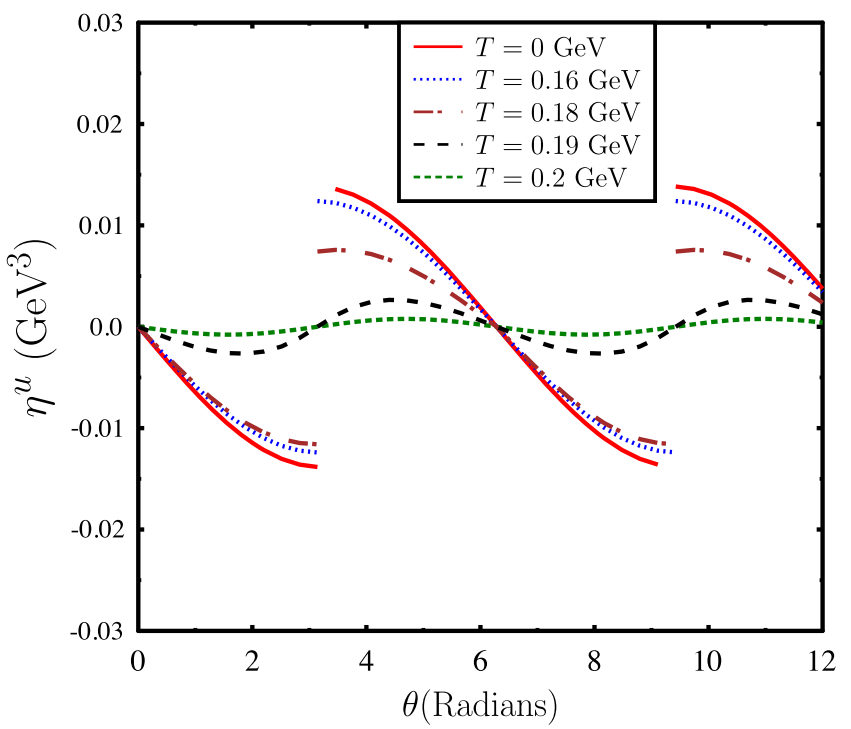

FIG. 5. Variation of $\eta^{u}$ with respect to $\theta$ for different values of $T$ in the PNJL model. At zero temperature $\eta$ has discontinuity at $\theta=(2 i+1) \pi$ and $\eta$ vanishes at $\theta=2 i \pi$, for $i=0,1,2 \ldots$ The presence of nonvanishing $\eta^{u}$ indicates breaking of the $C P$ symmetry. The degenerate vacua present at $\theta=(2 i+1) \pi$ are $C P$ conjugate. With increasing temperature this degenerate vacuum structure goes away. 


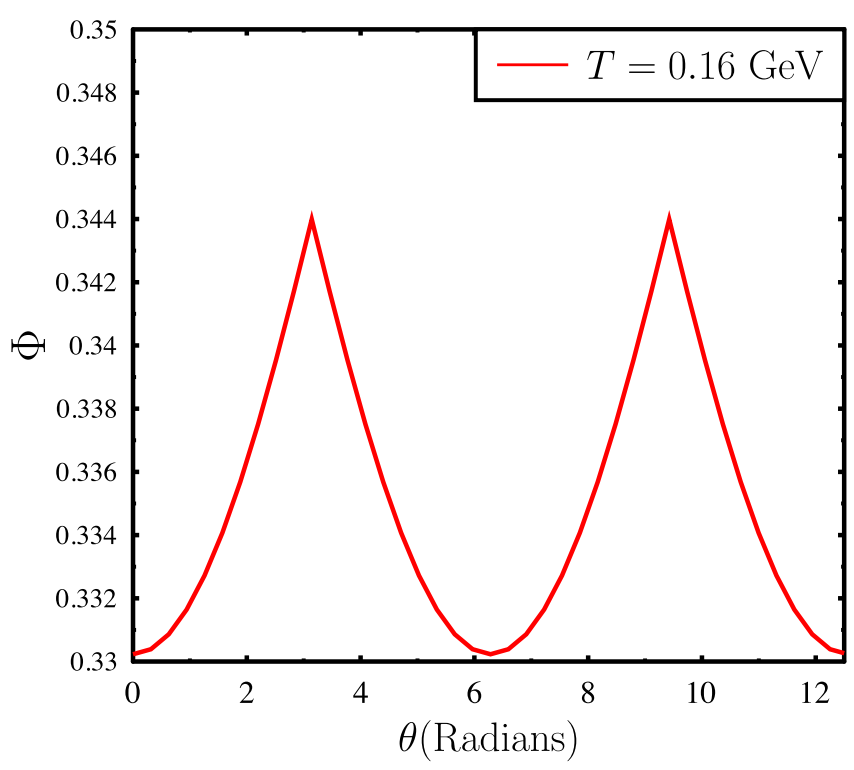

(a)

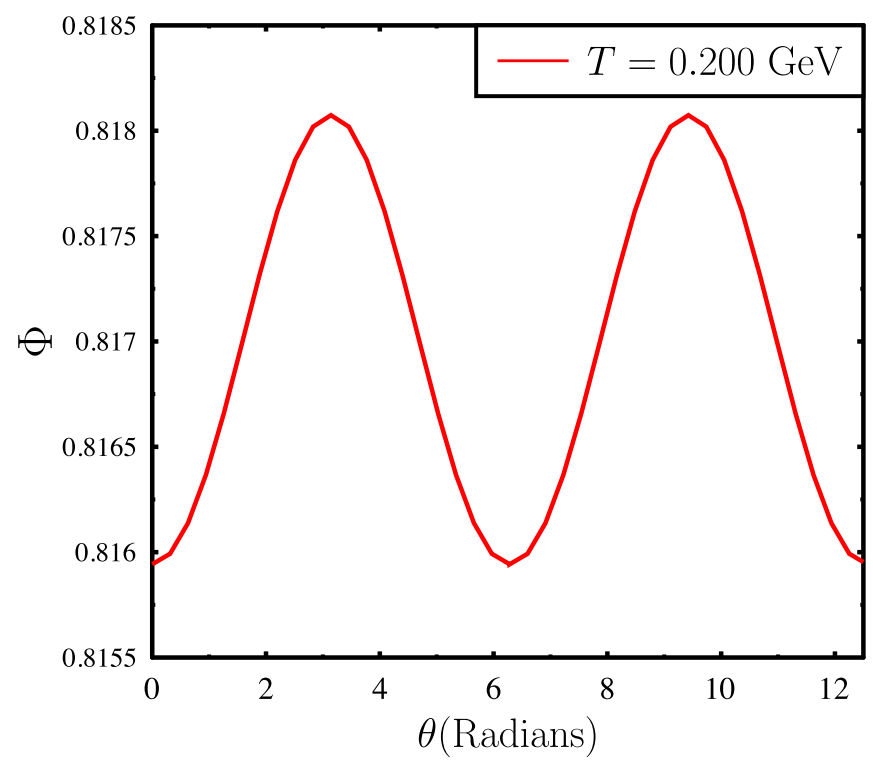

(b)

FIG. 6. (a) Variation of $\Phi$ with $\theta$ for $T=160 \mathrm{MeV}$. Variation of $\Phi$ with $\theta$ is similar to the variation of scalar condensate with $\theta$. Note that the Polyakov loop does not have direct coupling with the axion field. However due to gap equation $\Phi$ is connected with the other condensates which depend on $\theta$. Therefore $\Phi$ indirectly depends on $\theta$. (b) Variation of $\Phi$ with $\theta$ for $T=200 \mathrm{MeV}$.

$T_{c}$ and $\eta^{u}$ becomes a continuous function of $\theta$. The magnitude of $\eta^{u}$ decreases monotonically with an increase in temperature for all values of $\theta$.

In Fig. 6(a) we have shown the variation of Polyakov loop $\Phi$ with respect to $\theta$ at $T=160 \mathrm{MeV}$. As noted earlier, $\Phi$ becomes significant only near and beyond the critical temperature. The Polyakov loop variable $\Phi$ also oscillates with $\theta$ similar to scalar and pseudoscalar condensates.

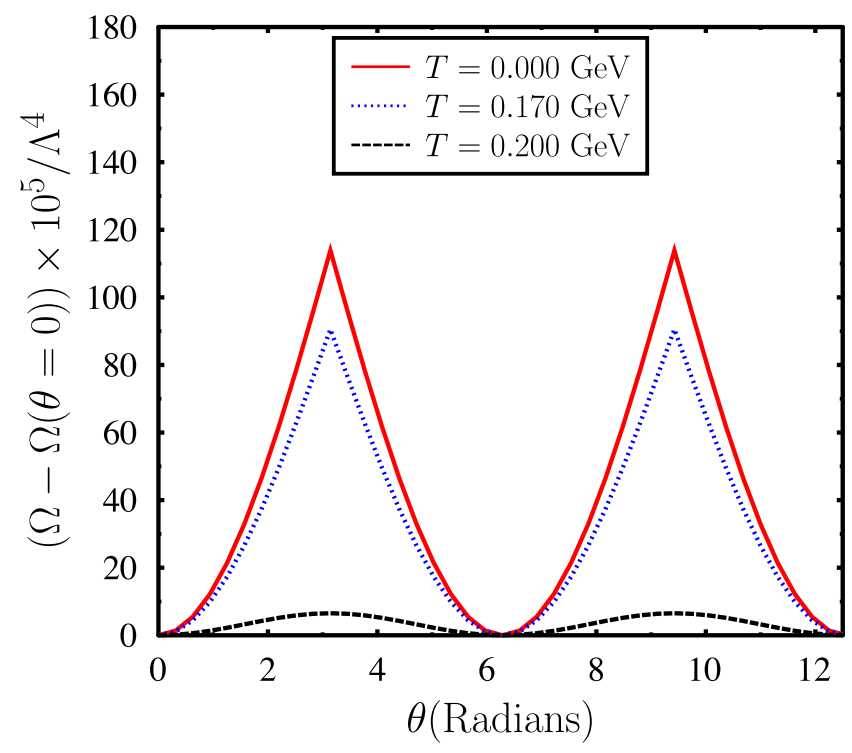

FIG. 7. Variation of the normalized thermodynamic potential for different values of the temperature in the PNJL model. The thermodynamic potential is given with respect to the potential at $\theta=0$ at each temperature.
However, the variation in $\Phi$ with $\theta$ is not significantly large. As mentioned earlier this insensitivity with $\theta$ is associated with the near constant value of the constituent quark masses with respect to $\theta$ as shown in Fig. 1(c). We find that such behavior of Polyakov loop variable $\Phi$ being almost independent of $\theta$ persists at higher temperature as seen in Fig. 6(b). Here the value of $\Phi$ is higher but the variation with $\theta$ is still negligible.

Figure 7 shows the variation of the normalized thermodynamic potential or the axion potential with respect to $\theta$ for different temperatures. At each temperature, the value of the thermodynamic potential at $\theta=0$ has been subtracted. This potential has degenerate vacua at $\theta=2 i \pi$ for $i=$ $0,1,2 \ldots$ etc. It also has maxima at $\theta=(2 i+1) \pi$. According to the Vafa-Witten theorem, the effective potential should have a minimum at $\theta=0$, which is clear from this figure. At finite temperature, the effective potential becomes flatter. As the temperature is increased from 0 to $170 \mathrm{MeV}$, which is below the transition temperature, the potential does not change much. This is because in this temperature range the condensate values do not change much in the PNJL model as shown in Figs. 1-3 respectively. Further for a higher temperature range above $170 \mathrm{MeV}$ the values of the different condensates, e.g., $\sigma, \eta$, and $\Phi$ change significantly in the PNJL model and so does the effective potential. As the temperature is increased further, the barrier height between degenerate vacua at $\theta=2 i \pi$ decreases. The barrier becomes negligibly small above $T_{c} \sim 188 \mathrm{MeV}$. One may also note that at $\theta=(2 i+1) \pi$, even though the pseudoscalar condensate vanishes above $T_{c}$, the thermodynamic potential is still maximum at $\theta=(2 i+1) \pi$. 

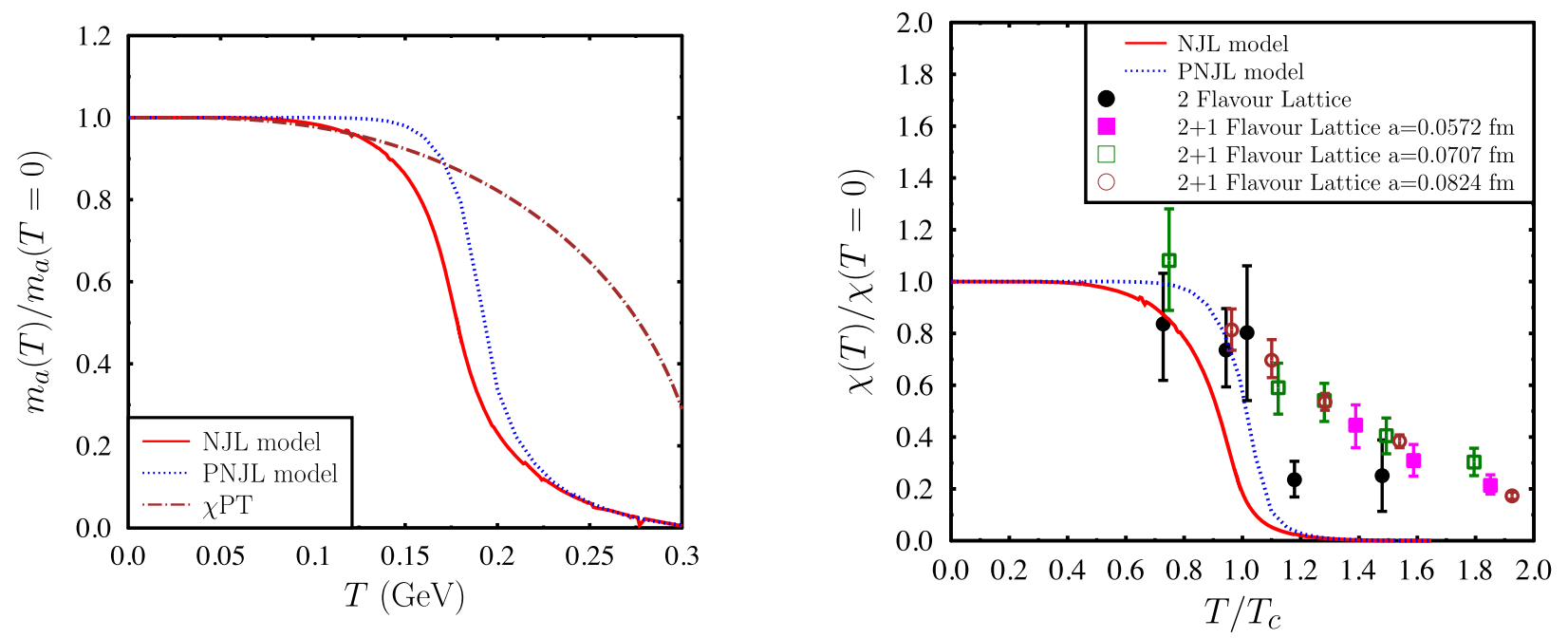

FIG. 8. Left plot: variation of the normalized axion mass $m_{a}(T) / m_{a}(T=0)$ with temperature for the PNJL model. $m_{a}(T=0)$ is the axion mass in vacuum. For comparison we have also plotted the NJL model results and chiral perturbation theory results for the axion mass. Right plot: variation of the topological susceptibility $\chi(T) / \chi(T=0)$ with temperature. For comparison we have also provided the results for the NJL model and 2 and $2+1$ flavor lattice QCD results as given in Ref. [111].

Next we show the variation of the ratio of axion mass $\left[m_{a}(T) / m_{a}(T=0)\right]$ at finite temperature normalized to its mass at zero temperature and the normalized topological susceptibility $[\chi(T) / \chi(T=0)]$ with temperature. In the left plot of Fig. 8 we show the variation of the normalized axion mass $\left[m_{a}(T) / m_{a}(T=0)\right]$ for the PNJL model. For comparison, we have given the normalized axion mass which is found in the NJL model and chiral perturbation theory $[70,71]$.

It may be relevant here to discuss the expression of the normalized topological susceptibility $\frac{\chi(T)}{\chi(T=0)}$ as may be estimated from chiral perturbation theory $(\chi \mathrm{PT})$ for three flavors [112]. The topological susceptibility is related to the quark condensate as [113]

$\chi=-\bar{m}^{2} \sum_{i} \frac{\left\langle\bar{q}_{i} q_{i}\right\rangle}{m_{i}}=\bar{m}^{2}\left(2 \frac{\sigma_{u}}{m_{u}}+\frac{\sigma_{s}}{m_{s}}\right), \quad \frac{1}{\bar{m}}=\sum_{i} \frac{1}{m_{i}}$.

We would like to comment here that, for the QCD vacuum, the condensates for the light flavors (including $u, d$, and $s$ quarks) are almost the same i.e., $\left\langle\bar{q}_{u} q_{u}\right\rangle \simeq$ $\left\langle\bar{q}_{d} q_{d}\right\rangle \simeq\left\langle\bar{q}_{s} q_{s}\right\rangle \equiv \sigma$ as also observed in lattice simulations [111]. In such a limit Eq. (24) reduces to the LeutwylerSmilga relation $\chi=\bar{m} \sigma$ at zero temperature [85]. However, this situation gets complicated at higher temperatures, particularly near the chiral restoration as the light quark condensate drops much faster than the strange quark condensate.

Indeed, this is, in fact, seen in Fig. 9 where we have plotted the ratio of $\sigma^{s} / \sigma^{u}$ estimated within the present PNJL model. Thus the estimation of temperature dependent topological susceptibility in three flavor $\chi \mathrm{PT}$ reduces to estimating the temperature dependent light $(u, d)$ and strange quark antiquark condensates. The leading temperature dependent contribution to the quark condensate is given by [114]

$$
\begin{gathered}
\sigma^{u}(T)=\left\langle\bar{q}_{u} q_{u}\right\rangle_{0}+\frac{\Sigma_{u}^{\pi}}{m_{u}} n_{\pi}+\frac{\Sigma_{u}^{K}}{m_{u}} n_{K}, \\
\sigma^{s}(T)=\left\langle\bar{q}_{s} q_{s}\right\rangle_{0}+\frac{\Sigma_{s}^{K}}{m_{s}} n_{K},
\end{gathered}
$$

where the scalar densities $n_{M}(M=\pi, K)$ of the Goldstone bosons are given as

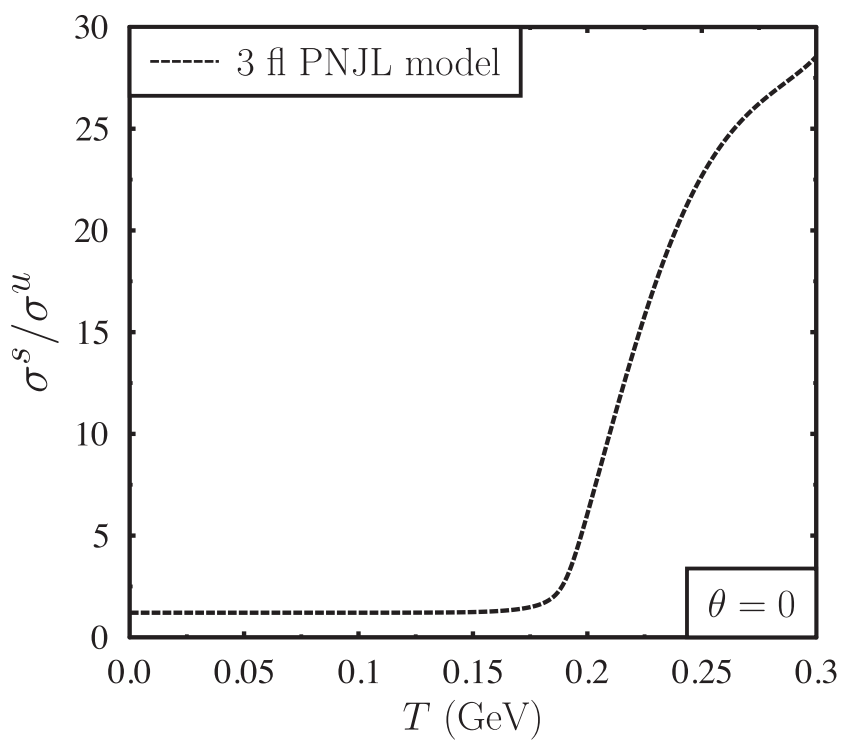

FIG. 9. Ratio of strange condensate to up quark condensate. 


$$
n_{M}=\frac{g_{M}}{(2 \pi)^{3}} \int d \mathbf{p} \frac{m_{M}}{E_{M}(\mathbf{p})} \frac{1}{\exp \left(\beta E_{M}(\mathbf{p})\right)-1}
$$

with $E_{M}(\mathbf{p})=\sqrt{\mathbf{p}^{2}+m_{M}^{2}}$ and $g_{M}$ is the corresponding degeneracy. Further, in the above $\Sigma_{i}^{M}$ is the mesonic sigma term that captures the response of the Goldstone boson masses to the changes in the current quark mass of a flavor $i$ and is given by

$$
\Sigma_{i}^{M}=m_{i} \frac{\partial m_{M}}{\partial m_{i}}
$$

The sigma terms for the pseudo-Goldstone bosons can be estimated by $\chi \mathrm{PT}$ using the Gell-Mann-Oakes-Renner relation at zero temperature [115]:

$$
f_{\pi}^{2} m_{\pi}^{2}\left(1-\kappa \frac{m_{\pi}^{2}}{f_{\pi}^{2}}\right)=\left(m_{u}+m_{d}\right) \sigma_{0}^{u}
$$

and

$$
f_{K}^{2} m_{K}^{2}\left(1-\kappa \frac{m_{K}^{2}}{f_{\pi}^{2}}\right)=\frac{\sigma_{0}^{u}+\sigma_{0}^{s}}{2}\left(m_{u}+m_{s}\right)
$$

These include the next to leading order correction incorporated through the parameter $\kappa=0.021$ [116]. Taking the derivatives with respect to current quark masses leads to the sigma terms for pion and kaon as

$$
\frac{\Sigma_{u}^{\pi}}{m_{u}}=\frac{\partial m_{\pi}}{\partial m_{u}}=\frac{\sigma_{0}^{u}}{f_{\pi}^{2} m_{\pi}}\left(1+2 \kappa \frac{m_{\pi}^{2}}{f_{\pi}^{2}}\right) \simeq \frac{m_{\pi}\left(1+\kappa \frac{m_{\pi}^{2}}{f_{\pi}^{2}}\right)}{4 m_{u}}
$$

and

$$
\frac{\Sigma_{u}^{K}}{m_{u}}=\frac{\Sigma_{s}^{K}}{m_{s}} \simeq \frac{m_{K}\left(1+\kappa \frac{m_{K}^{2}}{f_{\pi}^{2}}\right)}{2\left(m_{u}+m_{s}\right)} .
$$

We then have, using Eq. (24),

$$
\begin{aligned}
\frac{\chi(T)}{\chi(T=0)}= & 1-\frac{1}{2 f_{\pi}^{2}\left(1+\frac{C_{s}}{2} \frac{m_{u}}{m_{s}}\right)}\left[\left(\frac{n_{\pi}}{m_{\pi}}\right)\left(1+2 \kappa \frac{m_{\pi}^{2}}{f_{\pi}^{2}}\right)\right. \\
& +\left(\frac{n_{K}}{m_{K}}\right) \frac{1}{\left(f_{K} / f_{\pi}\right)^{2}}\left(1+2 \kappa \frac{m_{K}^{2}}{f_{\pi}^{2}}\right)\left(\frac{1+C_{s}}{2}\right) \\
& +\frac{1}{2}\left(\frac{m_{u}}{m_{s}}\right)\left(\frac{n_{K}}{m_{K}}\right) \frac{1}{\left(f_{K} / f_{\pi}\right)^{2}}\left(1+2 \kappa \frac{m_{K}^{2}}{f_{\pi}^{2}}\right) \\
& \left.\times\left(\frac{1+C_{s}}{2}\right)\right] .
\end{aligned}
$$

In the above equation $\sigma^{s}(T=0)=C_{s} \sigma^{u}(T=0)$. We have used here $f_{K} / f_{\pi}=1.22, C_{s}=0.8$ which gives $m_{u} / m_{s}=$ 0.041 [117].
Thus, the leading contribution arises from the first term in the square bracket and is given by

$$
\begin{aligned}
\frac{\chi(T)}{\chi(T=0)}= & 1-\frac{3}{4 \pi^{2}} \frac{T^{2}}{f_{\pi}^{2}} \int_{0}^{\infty} d q \frac{q^{2}}{\sqrt{q^{2}+\frac{m_{\pi}^{2}}{T^{2}}}} \\
& \times \frac{1}{\exp \left(\sqrt{q^{2}+\frac{m_{\pi}^{2}}{T^{2}}}\right)-1} \\
\equiv & 1-\frac{3 T^{2}}{f_{\pi}^{2}} J_{1}\left(m_{\pi}^{2} / T^{2}\right),
\end{aligned}
$$

which is exactly the two flavor result for the susceptibility [8]. Here,

$$
\begin{aligned}
& J_{n}(\zeta)=\frac{1}{(n-1) !}\left(-\frac{\partial}{\partial \zeta}\right)^{n} J_{0}(\zeta), \\
& J_{0}(\zeta)=-\frac{1}{\pi^{2}} \int_{0}^{\infty} d q q^{2} \log \left(1-\exp \left(-\sqrt{q^{2}+\zeta}\right)\right) .
\end{aligned}
$$

Note that according to Eq. (22), $\chi(T) / \chi(T=0)=m_{a}^{2}(T) /$ $m_{a}^{2}(T=0)$. Hence the topological susceptibility is just another way to represent the axion mass. The ratio of axion mass at finite temperature to axion mass at zero temperature is plotted in the left panel of Fig. 8. For comparison, we have plotted the same as obtained from the NJL model as well as the $\chi$ PT result of Eq. (33).

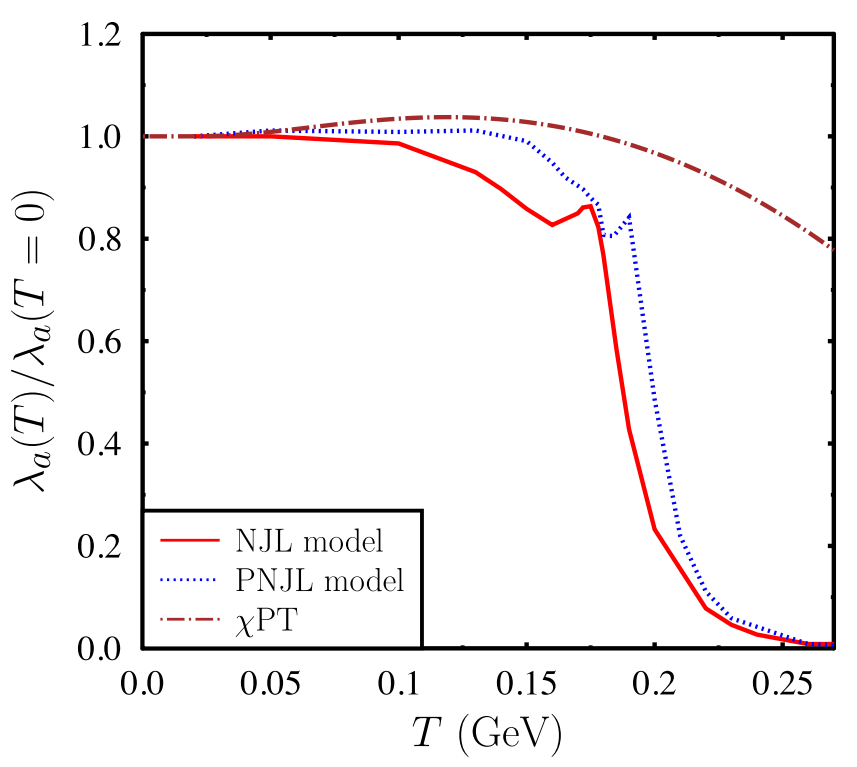

FIG. 10. Variation of the normalized axion self-coupling $\lambda_{a}(T) / \lambda_{a}(T=0)$ with temperature for the PNJL model. $\lambda_{a}(T=0)$ is the axion self-coupling in vacuum. For comparison we have also plotted the NJL model results and the chiral perturbation theory results for the axion self-coupling. 
It is important to note that the chiral perturbation theory results for topological susceptibility and axion masses are only applicable in the temperature range below the QCD transition scale. In the low-temperature range $(T \lesssim 100)$ $\mathrm{MeV}$ there is not much difference in the estimation of the axion mass for the NJL model, the PNJL model, and chiral perturbation theory. In fact, at zero temperature the axion mass as obtained in the PNJL model is $m_{a} f_{a}=6.07 \times 10^{-3} \mathrm{GeV}^{2}$, which is also the value of the axion mass obtained in the NJL model which is expected as Polyakov loop contribution is absent [71]. Note that the value of the axion mass at zero temperature as found in the NJL and PNJL model is similar to the other estimates of the axion mass, e.g., in lattice QCD [8] the value $m_{a} f_{a}$ is $5.7 \times 10^{-3} \mathrm{GeV}^{2}$. Only in the high-temperature range $(T>100 \mathrm{MeV})$, there is a significant difference between the results of the NJL and the PNJL model. This is because in the high-temperature range the Polyakov loop plays an important role in the PNJL model, where the scalar and the pseudoscalar condensate are affected by the Polyakov loop parameter $\Phi$. From this figure (Fig. 8), it is clear that the axion mass is sensitive to the chiral transition temperature. The increase in the chiral transition temperature for the PNJL model as compared to the NJL model is also manifested in the variation of the axion mass with temperature.

In the right plot of Fig. 8 we present the variation of the normalized topological susceptibility $\chi(T) / \chi(T=0)$ with $T / T_{c}$ in the PNJL model. For comparison, we have also given the results for the NJL model and three flavor lattice

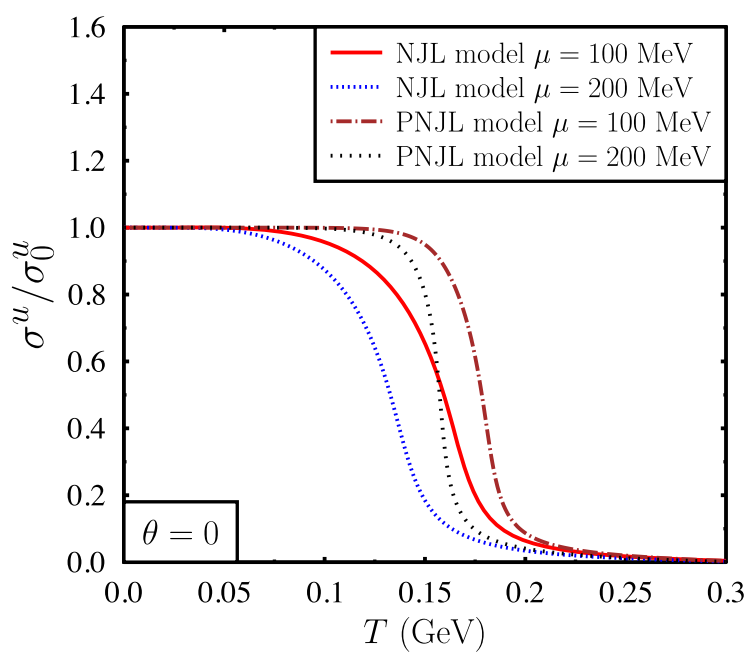

(a)

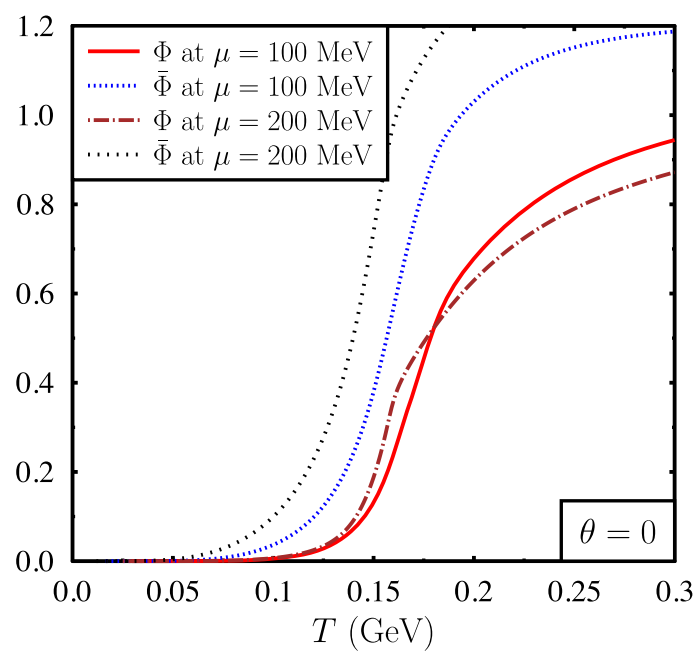

(b)

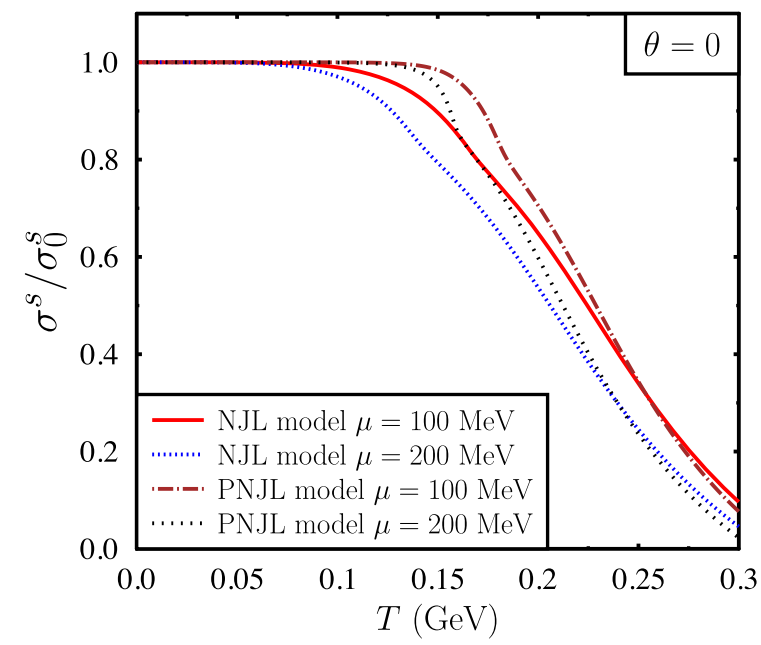

(c)

FIG. 11. (a) Variation of the normalized chiral condensate $\left(\sigma / \sigma_{0}\right)$ with temperature for nonvanishing values of the quark chemical potential. (b) Variation of the Polyakov loop $\Phi$, and its conjugate $\bar{\Phi}$ with temperature for nonvanishing values of the quark chemical potential. (c) Variation of normalized chiral condensate $\sigma^{s} / \sigma_{0}^{s}$ with temperature for nonzero chemical potential. 
QCD results [111]. Near and below the transition temperature PNJL model results for the normalized topological susceptibility are consistent with the lattice QCD results, however, one may note the NJL model results near the transition temperature are not in such a good agreement as in the PNJL model. Beyond $T_{c}$ there is a rather large discrepancy between the lattice and the PNJL model.

Variation of the normalized axion self-coupling $\lambda_{a}(T) / \lambda_{a}(T=0)$ with temperature is shown in Fig. 10. For comparison, we have also given the results for $\lambda_{a}(T) / \lambda_{a}(T=0)$ estimated in the NJL model and in the chiral perturbation theory $[70,71]$. In the low temperature range as compared to the QCD transition scale the temperature dependent axion self-coupling can be approximated by the expression for two flavor given as [8]

$$
\begin{aligned}
\frac{\lambda_{a}(T)}{\lambda_{a}}= & 1-\frac{3}{2} \frac{T^{2}}{f_{\pi}^{2}} J_{1}\left(\frac{m_{\pi}^{2}}{T^{2}}\right) \\
& +\frac{9}{2} \frac{m_{\pi}^{2}}{f_{\pi}^{2}} \frac{m_{u} m_{d}}{m_{u}^{2}-m_{u} m_{d}+m_{d}^{2}} J_{2}\left(\frac{m_{\pi}^{2}}{T^{2}}\right) .
\end{aligned}
$$

$J_{1}\left(\frac{m_{\pi}^{2}}{T^{2}}\right)$ and $J_{2}\left(\frac{m_{\pi}^{2}}{T^{2}}\right)$ in Eq. (36) can be obtained using Eq. (35).

At zero temperature the value of the axion self-coupling as estimated in the PNJL model is $\lambda_{a} f_{a}^{4}=-(53.71)^{4} \mathrm{MeV}^{4}$ which may be compared with results extrapolated from the lattice QCD estimation value of $\lambda_{a} f_{a}^{4}=-(57.9)^{4} \mathrm{MeV}^{4}$ [8]. It is clear from Fig. 10 that the results for the NJL model, chiral perturbation theory, and the PNJL model are in agreement in the low temperature range. But for the hightemperature range, the PNJL model results are significantly different from that of the NJL model results as well as $\chi \mathrm{PT}$.

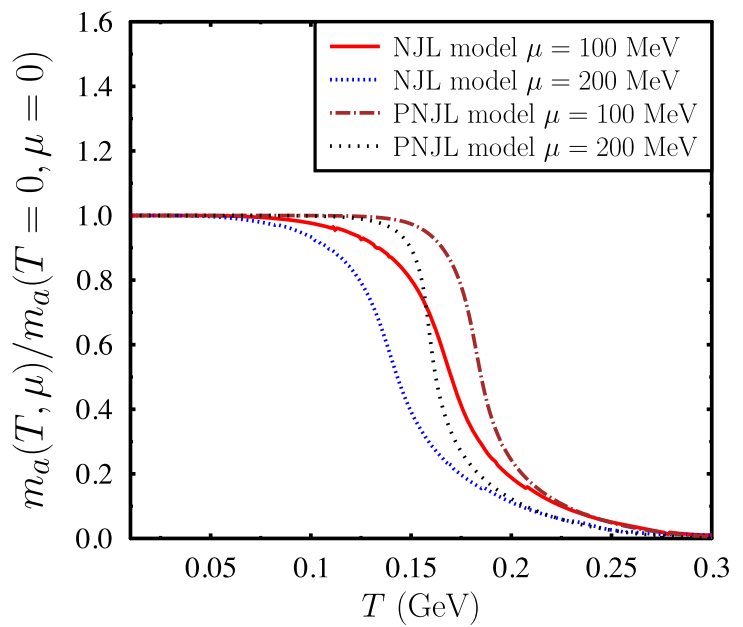

As mentioned earlier this is due to the fact that in the PNJL model the Polyakov loop $\Phi$ only becomes effective in the high-temperature range $T>100 \mathrm{MeV}$. The axion selfcoupling decreases with temperature and almost vanishes above the chiral crossover transition. We however observe a small peak near the transition temperature which we do not expect for any physical reason and could be a limitation of mean field approximation in evaluating the thermodynamic potential.

\section{B. Finite quark chemical potential}

In this subsection, we discuss the results for nonvanishing values of quark chemical potential $(\mu)$. In Fig. 11(a) we have shown the variation of the normalized chiral condensate $\left(\sigma^{u} / \sigma_{0}^{u}\right)$ for the light quarks. Figure 11(b) shows the variation of the Polyakov loop $\Phi$, and its conjugate $\bar{\Phi}$ with temperature for nonvanishing values of the quark chemical potential for $\theta=0$. Figure 11(a) indicates that with an increase in the quark chemical potential the chiral transition temperature decreases both in the NJL and the PNJL model. Note that for the range of quark chemical potential considered in these plots the chiral transition is smooth. Only at higher quark chemical potential and low temperature, one expects the chiral transition to be discontinuous [55]. From Fig. 11(b) we see that variation of the $\Phi$ and $\bar{\Phi}$ with the temperature at finite quark chemical potential is similar to the variation of $\Phi$ and $\bar{\Phi}$ at vanishing quark chemical potential. The only important difference here is the fact that, at finite quark chemical potential, the values of Polyakov loop $\Phi$ and its conjugate $\bar{\Phi}$ are not the same. In Fig. 11(c) we have plotted the same variation of chiral condensate for strange quark with the temperature at the finite chemical potential. The strange quark behaves in

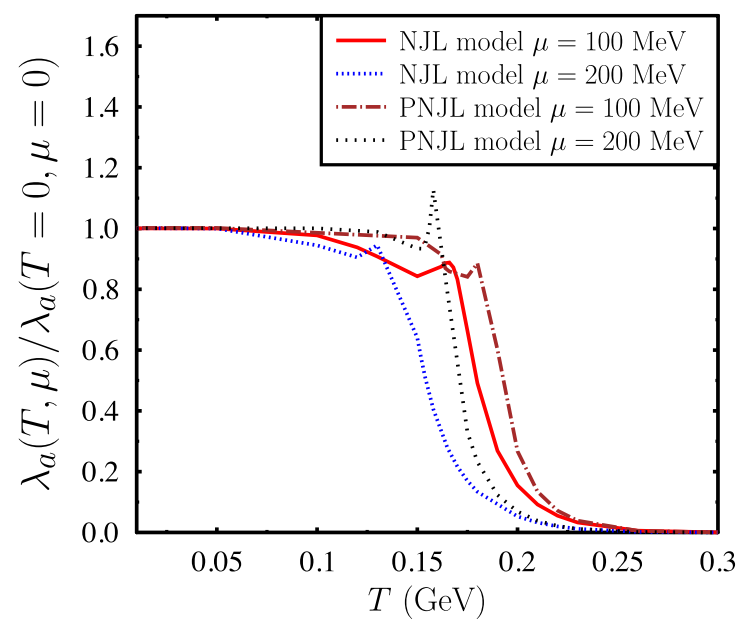

FIG. 12. Left plot: variation of the normalized axion mass at finite temperature and quark chemical potential with temperature for different values of the quark chemical potential. At finite quark chemical potential due to the suppression of the instanton effects the axion mass reduces with quark chemical potential. Right plot: variation of the normalized axion self-coupling at finite temperature and quark chemical potential, with temperature for different values of the quark chemical potential. Similar to the axion mass, axion selfcoupling also reduces with quark chemical potential. 


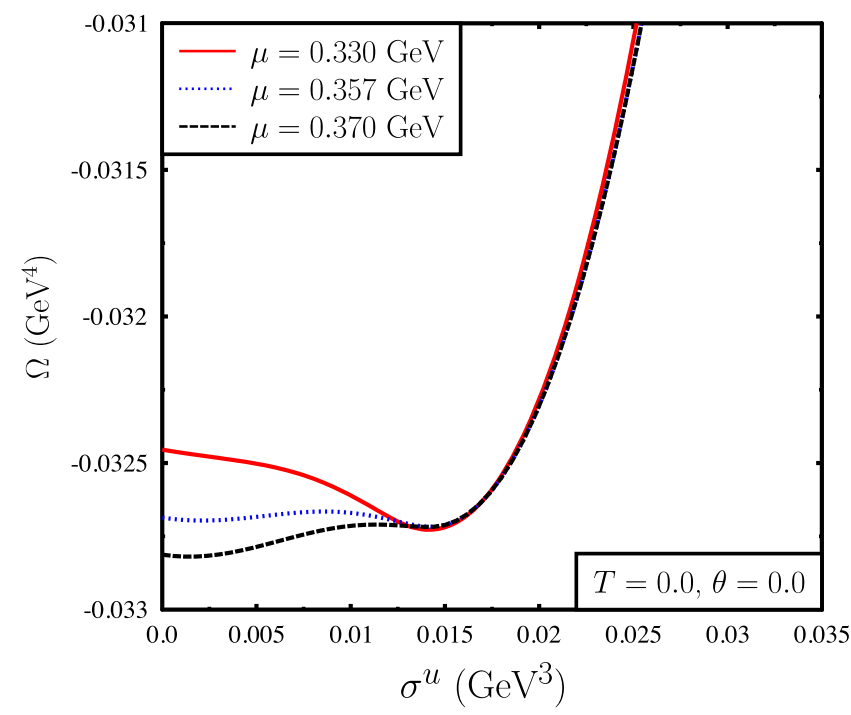

FIG. 13. Variation of the thermodynamic potential $\Omega$ with chiral condensate $\sigma$ at $T=0$ for different values of the quark chemical potential. Multiple vacuum structure of the thermodynamic potential is clearly observed in this figure. For $\mu \lesssim$ $0.357 \mathrm{GeV}$ the vacuum at $\sigma^{u} \sim 0.0144 \mathrm{GeV}^{3}$ is stable as compared to the vacuum at $\sigma \sim 0.00244 \mathrm{GeV}^{3}$. But for $\mu>$ $0.357 \mathrm{GeV}$ the vacuum at $\sigma \sim 0.00244 \mathrm{GeV}^{3}$ is stable. It is also clear that across the critical chemical potential the chiral condensate changes discontinuously. At zero temperature the chiral transition is first order in nature and the critical value of this transition is $\mu=0.357 \mathrm{GeV}$ for the parameters considered here.

a similar manner to light quarks, albeit its decrease with temperature and/or quark chemical potential is much slower compared to light quarks.

Next, we show the variation of the axion properties with temperature for nonvanishing values of the quark chemical potential. In the left plot of Fig. 12 we have shown the variation of the normalized axion mass with temperature

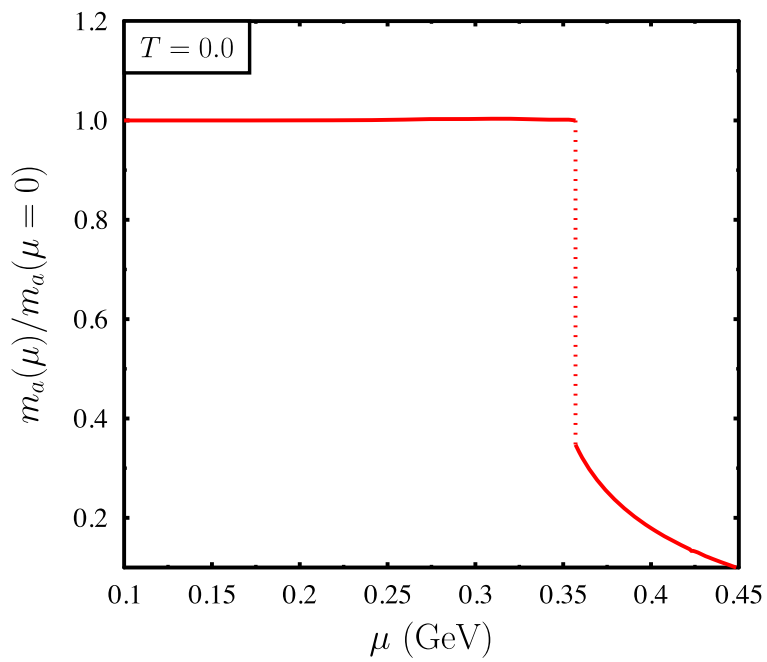

and quark chemical potential. It is clear that there is a correlation between the scalar condensate and the axion properties, i.e., in the chirally restored or deconfined phase the value of the scalar condensate, as well as the axion mass, is small with respect to the same in the confined or the chiral symmetry broken phase. With increasing quark chemical potential chiral transition temperature decreases which affects the axion mass. Hence with an increase in the quark chemical potential axion mass starts to decrease at a lower temperature both in the NJL and the PNJL model. In the right plot, we show the variation of the normalized axion self-coupling, i.e., $\lambda_{a}(T, \mu) / \lambda_{a}(T=0, \mu=0)$ with temperature and quark chemical potential. With an increase in the quark chemical potential, it is clear that $\lambda_{a}(T, \mu) / \lambda_{a}(T=0, \mu=0)$ starts to decrease at a lower temperature both in the NJL and the PNJL model. This behavior of $\lambda_{a}(T, \mu) / \lambda_{a}(T=0, \mu=0)$ is consistent with the fact that with an increase in the quark chemical potential chiral transition temperature decreases. It may be noted that similar to the case of vanishing chemical potential the quartic coupling shows a peak at the transition temperature which could be a limitation of the mean field potential.

It may be mentioned here that the effects of finite baryon density have also been recently discussed in Ref. [118]. At very high densities accessible inside the core of neutron stars the Fermi momentum is not far from the scale of QCD dynamics $\left(\Lambda_{\mathrm{QCD}}\right)$. In particular, for the chemical potential above the pion mass, finite density corrections can have an important impact on the properties of the axion [118]. It has been argued in Ref. [118] in the context of chiral Lagrangian extrapolated at finite density, that due to the suppression of the QCD-instantons the axion mass decreases in a dense medium with respect to the vacuum (without medium). We also get a suppression in the axion mass and coupling as estimated at finite quark chemical potential.

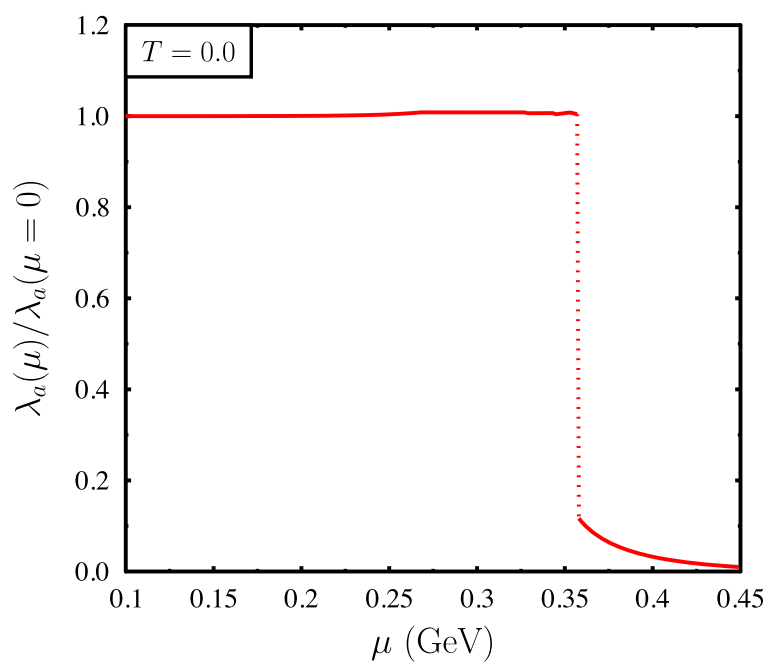

FIG. 14. Left plot: variation of the normalized axion mass $m_{a}(\mu) / m_{a}(\mu=0)$ with quark chemical potential $(\mu)$ at zero temperature. Right plot: variation of the normalized self-coupling of axion $\lambda_{a}(\mu) / \lambda_{a}(\mu=0)$ with quark chemical potential $(\mu)$ at zero temperature. Discontinuous behavior in the axion properties has been indicated by the dotted line. 
Finally, we discuss the results for vanishing temperature and finite quark chemical potential. In Fig. 13 we have shown the variation of the thermodynamic potential with the scalar condensate $\left(\sigma^{u}\right)$ for different values of quark chemical potential. For $\mu<357 \mathrm{MeV}$ the thermodynamic potential shows a minimum at $\sigma^{u}=0.0144 \mathrm{GeV}^{3}$. For $\mu=357 \mathrm{MeV}$ the effective potential has degenerate minima, one at $\sigma^{u}=0.00244 \mathrm{GeV}^{3}$ and $\sigma^{u}=0.0144 \mathrm{GeV}^{3}$ which defines the critical chemical potential $\mu_{c}$. Beyond $\mu=357 \mathrm{MeV}$ the position of minima changes discontinuously to the lower value of $\sigma^{u}$.

The discontinuous change of the chiral condensate also translates into the variation of the normalized mass of the axion $\left[m_{a}(\mu) / m_{a}(\mu=0)\right]$ and the axion self-coupling $\left[\lambda_{a}(\mu) / \lambda_{a}(\mu=0)\right]$. In the left plot of Fig. 14 we have shown that variation of the normalized mass of the axion $\left[m_{a}(\mu) / m_{a}(\mu=0)\right]$ with the quark chemical potential at zero temperature. On the other hand in the right plot of Fig. 14 we have shown that variation of the normalized axion self-coupling $\left[\lambda_{a}(\mu) / \lambda_{a}(\mu=0)\right]$ with the quark chemical potential at zero temperature. Due to the correlation between the chiral transition and the axion properties, we can see a discontinuous variation of the axion properties across the critical value of the quark chemical potential.

\section{CONCLUSION}

In the present investigation, we have studied the effects of hot and dense QCD medium on the axion properties e.g., its mass and self-coupling. In this investigation QCD medium at finite temperature and finite quark chemical potential has been modeled by Polyakov loop enhanced Nambu-Jona-Lasinio (PNJL) model for $(2+1)$ quark flavors. In Refs. [70,71] the temperature dependence of axion mass and self-coupling have been investigated within the framework of the Nambu-Jona-Lasinio model for two flavors. It turns out that for the three flavor PNJL model, the parity violating pseudoscalar condensate for strange quarks is an order of magnitude smaller than the same for the lighter $u, d$ quarks. Nonetheless, the strange quark condensates in the scalar channel affect the light quark condensates in the pseudoscalar channel significantly through the flavor mixing interaction and affect the $C P$ properties of the medium. Various methods have been used in literature to study the axion properties at finite temperature. For low temperature range, chiral perturbation theory $(\chi \mathrm{PT})$ gives a reliable prediction of the axion mass and selfcoupling. On the other hand at high temperature as compared to the QCD transition temperature perturbative techniques can be used to estimate the finite temperature effect on the axion mass and coupling. However, near the QCD transition scale where nonperturbative techniques are important one can use the QCD inspired effective models to describe the hot and dense QCD medium. The NJL model which is one of such QCD inspired models indicates that across the QCD transition temperature the axion properties can be significantly modified by the hot and dense medium. However, it is important to note that although the chiral transition or the phenomenological aspects of the chiral symmetry of QCD are incorporated in the NJL model, due to the lack of the QCD gauge fields, the confinement property of QCD is not properly incorporated in the NJL model. The Polyakov loop enhanced Nambu-Jona-Lasinio model, on the other hand, takes into account the phenomenological aspects of the chiral symmetry of QCD along with confinement in a unified framework. The Polyakov loop which takes a nonvanishing value at finite temperature and quark chemical potential plays an important role near the critical temperature in the PNJL model. Therefore there is a significant difference in axion properties calculated in the NJL and PNJL model due to the nonzero value of the Polyakov loop around the critical temperature. We find that axion properties are correlated with the chiral transition or confinement-deconfinement transition. The axion mass, topological susceptibility, self-coupling, etc., are significant up to the chiral transition temperature of $T \sim 188 \mathrm{MeV}$ in the PNJL model at vanishing quark chemical potential. These properties show a weak temperature dependence at low temperature as compared to the QCD transition temperature and they change significantly across the QCD transition scale. We have compared the normalized susceptibility (ratio of susceptibility at finite temperature to the susceptibility at zero temperature) at vanishing quark chemical potential with lattice QCD results. It matches reasonably well with lattice QCD results up to $T_{c}$. We have also calculated axion properties at a finite chemical potential. The critical temperature for chiral symmetry restoration decreases as chemical potential increases which also translates into the axion properties, i.e., axion mass and self-coupling decrease with chemical potential. At zero temperature but for finite quark chemical potential, different axion properties like mass and self-coupling are constant up to the critical chemical potential and decrease above the critical chemical potential. Axions are discussed in the literature in various contexts, e.g., early universe, dark matter, neutron stars, etc. It is important to note that in the context of the early universe the quark chemical potential or the baryon chemical potential is very small. Therefore in this case the effect of hot QCD medium on the axion properties can be an important input for the axion physics. On the other hand inside compact objects like neutron stars, the temperature is rather small but the baryon number density can be large. In this scenario, the effect of quark chemical potential on the axion properties can be significant in the study of axion physics.

\section{ACKNOWLEDGMENTS}

The work of A. D. is supported by the Polish National Science Center Grant No. 2018/30/E/ST2/00432. We thank the anonymous referee for checking the manuscript very carefully and making very important and useful suggestions. 
[1] R. D. Peccei and H. R. Quinn, Phys. Rev. Lett. 38, 1440 (1977).

[2] R. D. Peccei and H. R. Quinn, Phys. Rev. D 16, 1791 (1977).

[3] S. Weinberg, Phys. Rev. Lett. 40, 223 (1978).

[4] F. Wilczek, Phys. Rev. Lett. 40, 279 (1978).

[5] S. R. Coleman, Subnuclear series 15, 805 (1979), https:// inspirehep.net/literature/129404.

[6] R. J. Crewther, P. Di Vecchia, G. Veneziano, and E. Witten, Phys. Lett. 88B, 123 (1979); 91B, 487(E) (1980).

[7] J. M. Pendlebury et al., Phys. Rev. D 92, 092003 (2015).

[8] G. G. di Cortona, E. Hardy, J. P. Vega, and G. Villadoro, J. High Energy Phys. 01 (2016) 034.

[9] J. E. Kim and G. Carosi, Rev. Mod. Phys. 82, 557 (2010); 91, 049902(E) (2019).

[10] J. E. Kim, Phys. Rep. 150, 1 (1987).

[11] J. E. Kim, Phys. Rev. Lett. 43, 103 (1979).

[12] M. A. Shifman, A. I. Vainshtein, and V. I. Zakharov, Nucl. Phys. B166, 493 (1980).

[13] M. Dine, W. Fischler, and M. Srednicki, Phys. Lett. 104B, 199 (1981).

[14] A. Zhitnitsky, Sov. J. Nucl. Phys. 31, 260 (1980), https:// inspirehep.net/literature/157263.

[15] L. D. Duffy and K. van Bibber, New J. Phys. 11, 105008 (2009).

[16] M. S. Turner and F. Wilczek, Phys. Rev. Lett. 66, 5 (1991).

[17] L. Visinelli and P. Gondolo, Phys. Rev. D 80, 035024 (2009).

[18] G. G. Raffelt, Phys. Rep. 198, 1 (1990).

[19] M. S. Turner, Phys. Rep. 197, 67 (1990).

[20] H. Y. Cheng, Phys. Rep. 158, 1 (1988).

[21] J. E. Kim, Phys. Rep. 150, 1 (1987).

[22] D. J. Marsh, Phys. Rep. 643, 1 (2016).

[23] I.-S. Suh and C. H. Lee, Phys. Lett. B 432, 145 (1998).

[24] G. Raffelt and D. Seckel, Phys. Rev. Lett. 67, 2605 (1991).

[25] H.-T. Janka, W. Keil, G. Raffelt, and D. Seckel, Phys. Rev. Lett. 76, 2621 (1996).

[26] N. Iwamoto, Phys. Rev. Lett. 53, 1198 (1984).

[27] H. Umeda, N. Iwamoto, S. Tsuruta, L. Qin, and K. Nomoto, in Proceedings of the International Conference on Neutron Stars and Pulsars, Frontiers Science Series Vol. 24, edited by N. Shibazaki et al. (Universal Academy Press, 1998), p. 213, https://inspirehep.net/literature/454533.

[28] I. I. Tkachev, Phys. Lett. B 261, 289 (1991).

[29] E. W. Kolb and I. I. Tkachev, Phys. Rev. Lett. 71, 3051 (1993).

[30] P.-H. Chavanis, Phys. Rev. D 84, 043531 (2011).

[31] F. S. Guzman and L. A. Urena-Lopez, Astrophys. J. 645, 814 (2006).

[32] J. Barranco and A. Bernal, Phys. Rev. D 83, 043525 (2011).

[33] E. Braaten, A. Mohapatra, and H. Zhang, Phys. Rev. Lett. 117, 121801 (2016).

[34] S. Davidson and T. Schwetz, Phys. Rev. D 93, 123509 (2016).

[35] J. Eby, M. Leembruggen, P. Suranyi, and L. C. R. Wijewardhana, J. High Energy Phys. 12 (2016) 066.

[36] T. Helfer, D. J. Marsh, K. Clough, M. Fairbairn, E. A. Lim, and R. Becerril, J. Cosmol. Astropart. Phys. 03 (2017) 055.
[37] D. G. Levkov, A. G. Panin, and I. I. Tkachev, Phys. Rev. Lett. 118, 011301 (2017).

[38] J. Eby, M. Leembruggen, P. Suranyi, and L. C. R. Wijewardhana, J. High Energy Phys. 06 (2017) 014.

[39] L. Visinelli, S. Baum, J. Redondo, K. Freese, and F. Wilczek, Phys. Lett. B 777, 64 (2018).

[40] P. H. Chavanis, Phys. Rev. D 94, 083007 (2016).

[41] P. Sikivie, Int. J. Mod. Phys. A 25, 554 (2010).

[42] V. Desjacques, A. Kehagias, and A. Riotto, Phys. Rev. D 97, 023529 (2018).

[43] E. Berkowitz, M. I. Buchoff, and E. Rinaldi, Phys. Rev. D 92, 034507 (2015).

[44] S. Borsanyi, M. Dierigl, Z. Fodor, S. D. Katz, S. W. Mages, D. Nogradi, J. Redondo, A. Ringwald, and K. K. Szabo, Phys. Lett. B 752, 175 (2016).

[45] C. Bonati, M. DElia, M. Mariti, G. Martinelli, M. Mesiti, F. Negro, F. Sanfilippo, and G. Villadoro, J. High Energy Phys. 03 (2016) 155.

[46] J. Frison, R. Kitano, H. Matsufuru, S. Mori, and N. Yamada, J. High Energy Phys. 09 (2016) 021.

[47] S. Borsanyi et al., Nature (London) 539, 69 (2016).

[48] M. Dine, P. Draper, L. Stephenson-Haskins, and D. Xu, Phys. Rev. D 96, 095001 (2017).

[49] P. Fox, A. Pierce, and S. D. Thomas, arXiv:hep-th/ 0409059.

[50] M. P. Hertzberg, M. Tegmark, and F. Wilczek, Phys. Rev. D 78, 083507 (2008).

[51] P. Agrawal, G. Marques-Tavares, and W. Xue, J. High Energy Phys. 03 (2018) 049.

[52] S. Scherer, Adv. Nucl. Phys. 27, 277 (2003).

[53] U. G. Meissner, Rep. Prog. Phys. 56, 903 (1993).

[54] G. Ecker, Prog. Part. Nucl. Phys. 35, 1 (1995).

[55] M. Buballa, Phys. Rep. 407, 205 (2005).

[56] S. P. Klevansky, Rev. Mod. Phys. 64, 649 (1992).

[57] R. Brower, S. Chandrasekharan, J. W. Negele, and U. J. Wiese, Phys. Lett. B 560, 64 (2003).

[58] Y. Y. Mao and T. W. Chiu (TWQCD Collaboration), Phys. Rev. D 80, 034502 (2009).

[59] S. Aoki and H. Fukaya, Phys. Rev. D 81, 034022 (2010).

[60] V. Bernard, S. Descotes-Genon, and G. Toucas, J. High Energy Phys. 12 (2012) 080.

[61] V. Bernard, S. Descotes-Genon, and G. Toucas, J. High Energy Phys. 06 (2012) 051.

[62] M. A. Metlitski and A. R. Zhitnitsky, Phys. Lett. B 633, 721 (2006).

[63] S. Borsanyi, Z. Fodor, J. Guenther, K.-H. Kampert, S. D. Katz et al., Nature (London) 539, 69 (2016).

[64] M. Frank, M. Buballa, and M. Oertel, Phys. Lett. B 562 , 221 (2003).

[65] B. Chatterjee, H. Mishra, and A. Mishra, Phys. Rev. D 85, 114008 (2012).

[66] B. Chatterjee, H. Mishra, and A. Mishra, Phys. Rev. D 91, 034031 (2015).

[67] J. K. Boomsma and D. Boer, Phys. Rev. D 80, 034019 (2009).

[68] J. K. Boomsma and D. Boer, Phys. Rev. D 81, 074005 (2010).

[69] J. K. Boomsma and D. Boer, Phys. Rev. D 78, 054027 (2008). 
[70] Z.-Y. Lu and M. Ruggieri, Phys. Rev. D 100, 014013 (2019).

[71] A. Bandyopadhyay, R. L. S. Farias, B. S. Lopes, and R. O. Ramos, Phys. Rev. D 100, 076021 (2019).

[72] C. Ratti, S. Roessner, M. A. Thaler, and W. Weise, Eur. Phys. J. C 49, 213 (2007).

[73] C. Ratti, S. Roessner, and W. Weise, Phys. Lett. B 649, 57 (2007).

[74] C. Ratti, M. A. Thaler, and W. Weise, arXiv:nucl-th/ 0604025.

[75] C. Ratti, M. A. Thaler, and W. Weise, Phys. Rev. D 73, 014019 (2006).

[76] K. Fukushima, Phys. Rev. D 79, 074015 (2009).

[77] S. K. Ghosh, T. K. Mukherjee, M. G. Mustafa, and R. Ray, Phys. Rev. D 73, 114007 (2006).

[78] S. K. Ghosh, T. K. Mukherjee, M. G. Mustafa, and R. Ray, Phys. Rev. D 77, 094024 (2008).

[79] A. Bhattacharyya, P. Deb, S. K. Ghosh, and R. Ray, Phys. Rev. D 82, 014021 (2010).

[80] C. Ratti and W. Weise, Phys. Rev. D 70, 054013 (2004).

[81] A. M. Polyakov, Phys. Lett. 72B, 477 (1978).

[82] T. Bhattacharya, A. Gocksch, C. K. Altes, and R. D. Pisarski, Nucl. Phys. B383, 497 (1992).

[83] S. T. West and J.F. Wheater, Nucl. Phys. B486, 261 (1997).

[84] J. Boorstein and D. Kutasov, Phys. Rev. D 51, 7111 (1995).

[85] A. V. Smilga, Ann. Phys. (N.Y.) 234, 1 (1994).

[86] V. M. Belyaev, I. I. Kogan, G. W. Semenoff, and N. Weiss, Phys. Lett. B 277, 331 (1992).

[87] A. Dumitru and R. D. Pisarski, Phys. Lett. B 504, 282 (2001).

[88] A. Dumitru and R. D. Pisarski, Nucl. Phys. A698, 444 (2002).

[89] A. Dumitru and R. D. Pisarski, Phys. Rev. D 66, 096003 (2002).

[90] A. Dumitru, R. D. Pisarski, and D. Zschiesche, Phys. Rev. D 72, 065008 (2005).

[91] P. N. Meisinger, M. C. Ogilvie, and T. R. Miller, Phys. Lett. B 585, 149 (2004).

[92] G. G. Raffelt, Lect. Notes Phys. 741, 51 (2008).

[93] J. H. Chang, R. Essig, and S. D. McDermott, J. High Energy Phys. 09 (2018) 051.
[94] N. Bar, K. Blum, and G. Damico, Phys. Rev. D 101, 123025 (2020).

[95] A. Arvanitaki, S. Dimopoulos, S. Dubovsky, N. Kaloper, and J. March-Russell, Phys. Rev. D 81, 123530 (2010).

[96] A. Arvanitaki and S. Dubovsky, Phys. Rev. D 83, 044026 (2011).

[97] A. Arvanitaki, M. Baryakhtar, and X. Huang, Phys. Rev. D 91, 084011 (2015).

[98] C. Vafa and E. Witten, Phys. Rev. Lett. 53, 535 (1984).

[99] Y. Sakai, H. Kouno, T. Sasaki, and M. Yahiro, Phys. Lett. B 705, 349 (2011).

[100] P. D. Powell and G. Baym, Phys. Rev. D 85, 074003 (2012).

[101] E. Megias, E. R. Arriola, and L. L. Salecedo, Phys. Rev. D 74, 065005 (2006).

[102] B. W. Mintz, R. Stiele, R. O. Ramos, and J. S. Bielich, Phys. Rev. D 87, 036004 (2013).

[103] B.-J. Schaefer, J. M. Pawlowski, and J. Wambach, Phys. Rev. D 76, 074023 (2007).

[104] H. J. Warringa, D. Boer, and J. O. Anderson, Phys. Rev. D 72, 014015 (2005).

[105] T. Xia, L. He, and P. Zhuang, Phys. Rev. D 88, 056013 (2013).

[106] K. Fukushima, Phys. Lett. B 591, 277 (2004).

[107] S. Rößner, C. Ratti, and W. Weise, Phys. Rev. D 75, 034007 (2007).

[108] D. Gross, R. Pisarski, and L. Yaffe, Rev. Mod. Phys. 53, 43 (1981).

[109] D. Kharzeev, Ann. Phys. (Amsterdam) 325, 205 (2010).

[110] R. F. Dashen, Phys. Rev. D 3, 1879 (1971).

[111] B. Alles and M. D'Elia, arXiv:hep-lat/0602032; B. Alles, M. DElia, and A. Di Giacomo, Phys. Lett. B 483, 139 (2000).

[112] M. Kawaguchi, S. Matsuzaki, and A. Tomiya, Phys. Lett. B 813, 136044 (2021).

[113] Y. Y. Mao and T. W. Chiu, Phys. Rev. D 80, 034502 (2009).

[114] J. Jankowski, D. Blashke, and M. Spalinski, Phys. Rev. D 87, 105018 (2013).

[115] J. Gasser and H. Leutweler, Nucl. Phys. B250, 465 (1985).

[116] M. Jamin, Phys. Lett. B 538, 71 (2002).

[117] H. Lewtwyler and M. Roos, Z. Phys. C 25, 91 (1984).

[118] R. Balkin, J. Serra, K. Springmann, and A. Weiler, J. High Energy Phys. 07 (2020) 221. 